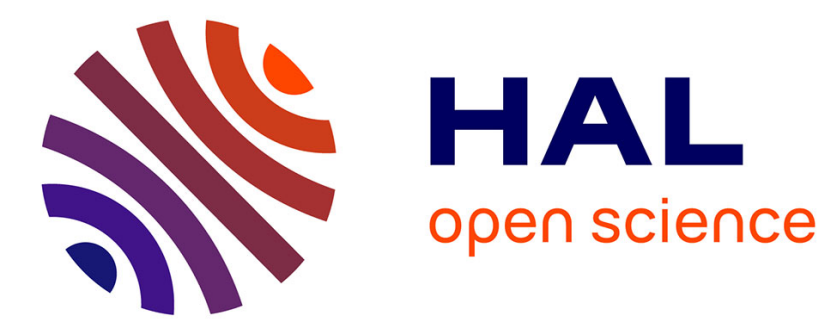

\title{
A LIOUVILLE PROPERTY WITH APPLICATION TO ASYMPTOTIC STABILITY FOR THE CAMASSA-HOLM EQUATION
}

Luc Molinet

\section{- To cite this version:}

Luc Molinet. A LIOUVILLE PROPERTY WITH APPLICATION TO ASYMPTOTIC STABILITY FOR THE CAMASSA-HOLM EQUATION. Archive for Rational Mechanics and Analysis, 2018, 230 (1), pp.185-230. 10.1007/s00205-018-1243-3 . hal-01768549

\section{HAL Id: hal-01768549 https://hal.science/hal-01768549}

Submitted on 17 Apr 2018

HAL is a multi-disciplinary open access archive for the deposit and dissemination of scientific research documents, whether they are published or not. The documents may come from teaching and research institutions in France or abroad, or from public or private research centers.
L'archive ouverte pluridisciplinaire HAL, est destinée au dépôt et à la diffusion de documents scientifiques de niveau recherche, publiés ou non, émanant des établissements d'enseignement et de recherche français ou étrangers, des laboratoires publics ou privés. 


\title{
A LIOUVILLE PROPERTY WITH APPLICATION TO ASYMPTOTIC STABILITY FOR THE CAMASSA-HOLM EQUATION
}

\author{
LUC MOLINET
}

\begin{abstract}
We prove a Liouville property for uniformly almost localized (up to translations) $H^{1}$-global solutions of the Camassa-Holm equation with a momentum density that is a non negative finite measure. More precisely, we show that such solution has to be a peakon.

As a consequence, we prove that peakons are asymptotically stable in the class of $H^{1}$-functions with a momentum density that belongs to $\mathcal{M}_{+}(\mathbb{R})$. Finally, we also get an asymptotic stability result for train of peakons.
\end{abstract}

\section{INTRODUCTION}

The Camassa-Holm equation $(\mathrm{C}-\mathrm{H})$,

$$
u_{t}-u_{t x x}=-3 u u_{x}+2 u_{x} u_{x x}+u u_{x x x}, \quad(t, x) \in \mathbb{R}^{2},
$$

can be derived as a model for the propagation of unidirectional shalow water waves over a flat bottom by writing the Green-Naghdi equations in Lie-Poisson Hamiltonian form and then making an asymptotic expansion which keeps the Hamiltonian structure ([9], [25]). A rigorous derivation of the Camassa-Holm equation from the full water waves problem is obtained in [1] and [15].

(C-H) is completely integrable (see [9],[10], [12] and [14]) and enjoys also a geometrical derivation (cf. [26], [27]). It possesses among others the following invariants

$M(v)=\int_{\mathbb{R}}\left(v-v_{x x}\right) d x, E(v)=\int_{\mathbb{R}} v^{2}(x)+v_{x}^{2}(x) d x$ and $F(v)=\int_{\mathbb{R}} v^{3}(x)+v(x) v_{x}^{2}(x) d x$

and can be written in Hamiltonian form as

$$
\partial_{t} E^{\prime}(u)=-\partial_{x} F^{\prime}(u)
$$

It is also worth noticing that (1.5) can be rewritted as

$$
y_{t}+u y_{x}+2 u_{x} y=0
$$

which is a transport equation for the momentum density $y=u-u_{x x}$.

Camassa and Holm [9] exhibited peaked solitary waves solutions to $(\mathrm{C}-\mathrm{H})$ that are given by

$$
u(t, x)=\varphi_{c}(x-c t)=c \varphi(x-c t)=c e^{-|x-c t|}, c \in \mathbb{R}^{*} .
$$

Date: April 17, 2018.

2010 Mathematics Subject Classification. 35Q35,35Q51, 35B40.

Key words and phrases. Camassa-Holm equation, asymptotic stability, peakon.

L.M. was partially supported by the french ANR project GEODISP. 
They are called peakon whenever $c>0$ and antipeakon whenever $c<0$. Note that the initial value problem associated with $(\mathrm{C}-\mathrm{H})$ has to be rewriten as

$$
\left\{\begin{array}{l}
u_{t}+u u_{x}+\left(1-\partial_{x}^{2}\right)^{-1} \partial_{x}\left(u^{2}+u_{x}^{2} / 2\right)=0 \\
u(0)=u_{0}
\end{array}\right.
$$

to give a meaning to these solutions.

Their stability seems not to enter the general framework developed for instance in [3], [23], especially because of the non smoothness of the peakon. However, Constantin and Strauss [18] succeeded in proving their orbital stability by a direct approach. This approach is based on two optimal inequalities: one involving $E(u-$ $\varphi)$ and $\max _{\mathbb{R}} u$ and the other one involving $E(u), F(u)$ and $\max _{\mathbb{R}} u$.

In a series of papers (see for instance [29], [30]) Martel and Merle developped an approach, based on a Liouville property for uniformly almost localized global solutions close to the solitary waves, to prove the asymptotic stability for a wide class of dispersive equations. The Liouville property is based on the study of a dual equation related to the linearized equation around the solitary waves. Such approach to prove the Liouville property seems not to be applicable for the $\mathrm{C}-\mathrm{H}$ equation. Indeed, first working with the dual problem requires more regularity on the solution and, in contrast to $\mathrm{KdV}$-like equations, one cannot require the asymptotic objects of the $\mathrm{C}-\mathrm{H}$ equation to be smooth (see the peakon!). Second, for the same reasons for which there is no proof of the orbital stability by the spectral method, it seems very difficult to get a non negative property on the underlying linear operator.

In this paper we prove a Liouville result for uniformly almost localized (up to translations) global solutions to the $\mathrm{CH}$ equation and then follows the general strategy developed by Martel and Merle to deduce the asymptotic stability of the peakon. The main ingredient to prove our Liouville result is the finite speed propagation of the momentum density of the solution. We would like to underline that our arguments are not specific to the Camassa-Holm equation but can be adapted for a wide class of equations with peakons as we will show in a forthcoming work.

In this paper we will work in the framework of the solutions constructed in [17]. This class corresponds to solutions emanating from initial data that belong to $H^{1}(\mathbb{R})$ with a density momentum that is a non negative finite measure. It has the advantage to contain the peakon and to enjoy good properties as global existence, uniqueness and $H^{1}$-continuity of the flow.

It is worth noticing that, recently ${ }^{1}$, Bressan and Constantin ([6], [7]) succeed to construct global conservative and dissipative solutions of the (1.5) for initial data in $H^{1}(\mathbb{R})$ by using scalar conservation laws techniques. The uniqueness of the conservative solution has been shown very recently by Bressan-Chen-Zhang [5] but its continuity with values in $H^{1}(\mathbb{R})$ is not known. In consequence, the orbital stability of the peakon with respect to these solutions is still an open problem. In this direction, note that a Lipschitz metric on $H^{1}$-bounded sets has been very recently constructed in [8].

Before stating our results let us introduce the function space to which our initial data belong. Following [17], we introduce the following space of functions

$$
Y=\left\{u \in H^{1}(\mathbb{R}) \text { such that } u-u_{x x} \in \mathcal{M}(\mathbb{R})\right\}
$$

\footnotetext{
${ }^{1}$ See for instance [31] for a survey on previous existence results.
} 
where $\mathcal{M}(\mathbb{R})$ is the space of finite Radon measures on $\mathbb{R}$. We denote by $Y_{+}$the closed subset of $Y$ defined by $Y_{+}=\left\{u \in Y / u-u_{x x} \in \mathcal{M}_{+}\right\}$where $\mathcal{M}_{+}$is the set of non negative finite Radon measures on $\mathbb{R}$.

Let $C_{b}(\mathbb{R})$ be the set of bounded continuous functions on $\mathbb{R}, C_{0}(\mathbb{R})$ be the set of continuous functions on $\mathbb{R}$ that tends to 0 at infinity and let $I \subset \mathbb{R}$ be an interval. A sequence $\left\{\nu_{n}\right\} \subset \mathcal{M}$ is said to converge tightly (resp. weakly) towards $\nu \in \mathcal{M}$ if for any $\phi \in C_{b}(\mathbb{R})\left(\operatorname{resp} . C_{0}(\mathbb{R})\right),\left\langle\nu_{n}, \phi\right\rangle \rightarrow\langle\nu, \phi\rangle$. We will then write $\nu_{n} \rightarrow * \nu$ tightly in $\mathcal{M}$ (resp. $\nu_{n} \rightarrow * \nu$ in $\left.\mathcal{M}\right)$.

Throughout this paper, $y \in C_{t i}(I ; \mathcal{M})$ (resp. $y \in C_{w}(I ; \mathcal{M})$ ) will signify that for any $\phi \in C_{b}(\mathbb{R})\left(\right.$ resp. $\left.\phi \in C_{0}(\mathbb{R})\right), t \mapsto\langle y(t), \phi\rangle$ is continuous on $I$ and $y_{n} \rightarrow * y$ in $C_{t i}(I ; \mathcal{M})$ (resp. $y_{n} \rightarrow * y$ in $C_{w}(I ; \mathcal{M})$ ) will signify that for any $\phi \in C_{b}(\mathbb{R})$ (resp. $\left.C_{0}(\mathbb{R})\right),\left\langle y_{n}(\cdot), \phi\right\rangle \rightarrow\langle y(\cdot), \phi\rangle$ in $C(I)$.

Definition 1.1. We say that a solution $u \in C\left(\mathbb{R} ; H^{1}(\mathbb{R})\right)$ with $u-u_{x x} \in C_{w}\left(\mathbb{R} ; \mathcal{M}_{+}\right)$ of (1.5) is $Y$-almost localized if there exist $c>0$ and a $C^{1}$-function $x(\cdot)$, with $\dot{x} \geq c>0$, for which for any $\varepsilon>0$, there exists $R_{\varepsilon}>0$ such that for all $t \in \mathbb{R}$ and all $\Phi \in C(\mathbb{R})$ with $0 \leq \Phi \leq 1$ and $\operatorname{supp} \Phi \subset\left[-R_{\varepsilon}, R_{\varepsilon}\right]^{c}$.

$$
\int_{\mathbb{R}}\left(u^{2}(t)+u_{x}^{2}(t)\right) \Phi(\cdot-x(t)) d x+\left\langle\Phi(\cdot-x(t)), u(t)-u_{x x}(t)\right\rangle \leq \varepsilon .
$$

Theorem 1.1. Let $u \in C\left(\mathbb{R} ; H^{1}(\mathbb{R})\right)$, with $u-u_{x x} \in C_{w}\left(\mathbb{R} ; \mathcal{M}_{+}\right)$, be a $Y$-almost localized solution of (1.5) that is not identically vanishing. Then there exists $c^{*}>0$ and $x_{0} \in \mathbb{R}$ such that

$$
u(t)=c^{*} \varphi\left(\cdot-x_{0}-c^{*} t\right), \quad \forall t \in \mathbb{R} .
$$

Remark 1.1. This theorem implies, in particular, that a $Y$-almost localized solution with non negative momentum density cannot be smooth for any time. More precisely, if $u \in C\left(\mathbb{R} ; H^{1}\right)$, with $u-u_{x x} \in C_{w}\left(\mathbb{R} ; \mathcal{M}_{+}\right)$, is a $Y$-almost localized solution of the Camassa-Holm equation that belongs to $H^{\frac{3}{2}}(\mathbb{R})$ for some $t \in \mathbb{R}$ then $u$ must be the trivial null solution.

As a consequence we get the asymptotic stablity of the peakons :

Theorem 1.2. Let $c>0$ be fixed. There exists a universal constant $0<\eta_{0} \ll 1$ such that for any $0<\theta<c$ and any $u_{0} \in Y_{+}$satisfying

$$
\left\|u_{0}-\varphi_{c}\right\|_{H^{1}} \leq \eta_{0}\left(\frac{\theta}{c}\right)^{8}
$$

there exists $c^{*}>0$ with $\left|c-c^{*}\right| \ll c$ and a $C^{1}$-function $x: \mathbb{R} \rightarrow \mathbb{R}$ with $\lim _{t \rightarrow \infty} \dot{x}=c^{*}$ such that

$$
u(t, \cdot+x(t)) \underset{t \rightarrow+\infty}{\rightarrow} \varphi_{c^{*}} \text { in } H^{1}(\mathbb{R}),
$$

where $u \in C\left(\mathbb{R} ; H^{1}\right)$ is the solution emanating from $u_{0}$. Moreover,

$$
\lim _{t \rightarrow+\infty}\left\|u(t)-\varphi_{c^{*}}(\cdot-x(t))\right\|_{H^{1}(] \theta t,+\infty[)}=0 .
$$

Remark 1.2. Using that (1.5) is invariant by the change of unknown $u(t, x) \mapsto$ $-u(t,-x)$, we obtain as well the asymptotic stability of the antipeakon profile $c \varphi$ with $c<0$ in the class of $H^{1}$-function with a momentum density that belongs to $\mathcal{M}_{-}(\mathbb{R})$. 
Remark 1.3. This theorem implies the growth of the high Sobolev norms for some smooth solutions of the Camassa-Holm equation. Indeed, it is proven in [13] that any initial datum $u_{0} \in H^{\infty}(\mathbb{R}) \cap Y_{+}$gives rise to a solution $u \in C\left(\mathbb{R} ; H^{\infty}(\mathbb{R})\right)$ and the above theorem ensures that if such initial datum satisfies (1.8), then $\|u(t)\|_{H^{3 / 2}} \rightarrow+\infty$ as $t \rightarrow+\infty$.

Remark 1.4. Theorem 1.2 and especially Theorem 6.2, in Section 6, are first steps towards the peakons decomposition of solutions with a non negative momentum density that is studied by the inverse scattering approach in [19].

This paper is organized as follows : in the next section we recall the wellposedness results for the class of solutions we will work with and, in Section 3, we derive an almost monotonicity result that implies an exponential decay result for $Y$-almost localized global solutions. Section 4 is devoted to the Liouville theorem for $Y$-almost localized solutions which is the heart of this work. Finally, in Section 5 and 6 , we respectively prove the asymptotic stability of a single peakon and of a train of peakons.

\section{Global well-Posedness Results}

We first recall some obvious estimates that will be useful in the sequel of this paper. Noticing that $p(x)=\frac{1}{2} e^{-|x|}$ satisfies $p * y=\left(1-\partial_{x}^{2}\right)^{-1} y$ for any $y \in H^{-1}(\mathbb{R})$ we easily get

$$
\|u\|_{W^{1,1}}=\left\|p *\left(u-u_{x x}\right)\right\|_{W^{1,1}} \lesssim\left\|u-u_{x x}\right\|_{\mathcal{M}}
$$

and

$$
\left\|u_{x x}\right\|_{\mathcal{M}} \leq\|u\|_{L^{1}}+\left\|u-u_{x x}\right\|_{\mathcal{M}}
$$

which ensures that

$$
Y \hookrightarrow\left\{u \in W^{1,1}(\mathbb{R}) \text { with } u_{x} \in \mathcal{B} V(\mathbb{R})\right\} .
$$

It is also worth noticing that since for $v \in C_{0}^{\infty}(\mathbb{R})$,

$$
v(x)=\frac{1}{2} \int_{-\infty}^{x} e^{x^{\prime}-x}\left(v-v_{x x}\right)\left(x^{\prime}\right) d x^{\prime}+\frac{1}{2} \int_{x}^{+\infty} e^{x-x^{\prime}}\left(v-v_{x x}\right)\left(x^{\prime}\right) d x^{\prime}
$$

and

$$
v_{x}(x)=-\frac{1}{2} \int_{-\infty}^{x} e^{x^{\prime}-x}\left(v-v_{x x}\right)\left(x^{\prime}\right) d x^{\prime}+\frac{1}{2} \int_{x}^{+\infty} e^{x-x^{\prime}}\left(v-v_{x x}\right)\left(x^{\prime}\right) d x^{\prime},
$$

we get $v_{x}^{2} \leq v^{2}$ as soon as $v-v_{x x} \geq 0$ on $\mathbb{R}$. By the density of $C_{0}^{\infty}(\mathbb{R})$ in $Y$, we deduce that

$$
\left|v_{x}\right| \leq v \text { for any } v \in Y_{+} .
$$

Finally, throughout this paper, we will denote $\left\{\rho_{n}\right\}_{n \geq 1}$ the mollifer defined by

$$
\rho_{n}=\left(\int_{\mathbb{R}} \rho(\xi) d \xi\right)^{-1} n \rho(n \cdot) \text { with } \rho(x)=\left\{\begin{array}{lll}
e^{1 /\left(x^{2}-1\right)} & \text { for } & |x|<1 \\
0 & \text { for } & |x| \geq 1
\end{array}\right.
$$

In [13] a global well-posedness result is shown for smooth solution to (1.5) with a non negative momentum density. This result can be summarized in the following proposition 
Proposition 2.1. (Global smooth solutions [13])

Let $u_{0} \in H^{3}(\mathbb{R})$, satisfying $y_{0}=u_{0}-u_{0, x x} \geq 0$ with $y_{0} \in L^{1}(\mathbb{R})$, then the initial value problem associated with $(1.5)$ has a unique solution $u \in C\left(\mathbb{R} ; H^{3}(\mathbb{R})\right) \cap$ $C^{1}\left(\mathbb{R} ; H^{2}(\mathbb{R})\right)$. This solution satisfies $y=u-u_{x x} \geq 0$ on $\mathbb{R}^{2}$ and $M(\cdot), E(\cdot)$ and $F(\cdot)$ are constant along the trajectory ${ }^{2}$. If moreover $u_{0} \in H^{\infty}(\mathbb{R})$ then $u \in C\left(\mathbb{R} ; H^{\infty}(\mathbb{R})\right)$.

Unfortunately, the peakons do not enter in this framework since their profiles do not belong even to $H^{\frac{3}{2}}(\mathbb{R})$. In [17] an existence and uniqueness result of global solutions to (1.5) in a class of functions that contains the peakon is proved. This result will be crucial in our analysis. We give below a slightly improved version:

Proposition 2.2. (Global weak solution [17])

Let $u_{0} \in Y_{+}$be given.

1. Uniqueness and global existence : (1.5) has a unique solution $u \in C^{1}\left(\mathbb{R} ; L^{2}(\mathbb{R})\right) \cap$ $C\left(\mathbb{R} ; H^{1}(\mathbb{R})\right)$ such that $y=\left(1-\partial_{x}^{2}\right) u \in C_{t i}\left(\mathbb{R} ; \mathcal{M}_{+}\right)$. Moreover, $E(u) F(u)$ and $M(u)=\langle y, 1\rangle$ are conservation laws .

2. Continuity with respect to initial data in $H^{1}(\mathbb{R})$ : For any sequence $\left\{u_{0, n}\right\}$ bounded in $Y_{+}$such that $u_{0, n} \rightarrow u_{0}$ in $H^{1}(\mathbb{R})$ and $\left(1-\partial_{x}^{2}\right) u_{0, n} \rightarrow * u_{0}-u_{0, x x}$ tightly in $\mathcal{M}$, the emanating sequence of solution $\left\{u_{n}\right\} \subset C^{1}\left(\mathbb{R}_{+} ; L^{2}(\mathbb{R})\right) \cap C\left(\mathbb{R}_{+} ; H^{1}(\mathbb{R})\right)$ satisfies for any $T>0$

$$
u_{n} \rightarrow u \text { in } C\left([-T, T] ; H^{1}(\mathbb{R})\right)
$$

and

$$
\left(1-\partial_{x}^{2}\right) u_{n} \rightarrow * y \text { in } C_{t i}([-T, T], \mathcal{M}) \text {. }
$$

3. Continuity with respect to initial data in $Y$ equipped with the weak topology: For any sequence $\left\{u_{0, n}\right\} \subset Y_{+}$such that $u_{0, n} \rightarrow * u_{0}$ in $Y$, the emanating sequence of solution $\left\{u_{n}\right\} \subset C^{1}\left(\mathbb{R} ; L^{2}(\mathbb{R})\right) \cap C\left(\mathbb{R}_{+} ; H^{1}(\mathbb{R})\right)$ satisfies for any $T>0$,

$$
u_{n} \underset{n \rightarrow \infty}{\rightarrow} \text { in } C_{w}\left([-T, T] ; H^{1}(\mathbb{R})\right),
$$

and

$$
\left(1-\partial_{x}^{2}\right) u_{n} \rightarrow * y \text { in } C_{w}([-T, T], \mathcal{M}) .
$$

Proof. The uniqueness and global existence results are obtained in [17] except the conservation of $M(u)$ and the fact that $y$ belongs to $C_{t i}\left(\mathbb{R} ; \mathcal{M}_{+}\right)$. In [17], only the fact that $y \in L^{\infty}\left(\mathbb{R}, \mathcal{M}_{+}\right)$is stated. Note also that in [17] the results are stated only for positive times but, since the equation is reversible with time, it is direct to check that the results hold as well for negative times.

To prove (2.4), it suffices to notice that, according to the conservation of the $H^{1}$ norm and (2.1), the sequence of emanating solution $\left\{u_{n}\right\}$ is bounded in $C\left(\mathbb{R}_{+} ; H^{1}(\mathbb{R})\right) \cap$ $L^{\infty}\left(\mathbb{R} ; W^{1,1}(\mathbb{R})\right)$ with $\left\{u_{n, x}\right\}$ bounded in $L^{\infty}(\mathbb{R} ; \mathcal{B} V(\mathbb{R}))$. Therefore, there exists $v \in L^{\infty}\left(\mathbb{R} ; H^{1}(\mathbb{R})\right)$ with $\left(1-\partial_{x}^{2}\right) v \in L^{\infty}\left(\mathbb{R} ; \mathcal{M}_{+}(\mathbb{R})\right)$ and an increasing sequence of integers $\left\{n_{k}\right\}_{k \geq 1}$ such that, for any $T>0$,

$u_{n_{k}} \underset{k \rightarrow \infty}{\rightarrow} v \in L^{\infty}\left([-T, T] ; H^{1}(\mathbb{R})\right)$ and $\left(1-\partial_{x}^{2}\right) u_{n_{k}} \underset{k \rightarrow \infty}{\rightarrow} *\left(1-\partial_{x}^{2}\right) v$ in $L^{\infty}(]-T, T\left[; \mathcal{M}_{+}(\mathbb{R})\right)$

\footnotetext{
${ }^{2}$ In particular, $y \in L^{\infty}\left(\mathbb{R} ; L^{1}(\mathbb{R})\right)$.

${ }^{3} \mathrm{By}$ this we mean that $u_{0, n} \rightarrow u_{0}$ in $H^{1}(\mathbb{R})$ and $\left(1-\partial_{x}^{2}\right) u_{0, n} \rightarrow * u_{0}-u_{0, x x}$ in $\mathcal{M}$
} 
But, using that $\left\{\partial_{t} u_{n}\right\}$ is bounded in $L^{\infty}\left(\mathbb{R} ; L^{2}(\mathbb{R}) \cap L^{1}(\mathbb{R})\right)$, Helly's, Aubin-Lions' compactness and Arzela-Ascoli's theorems then ensure that $v$ is a solution to (1.5) that belongs to $C_{w}\left([-T, T] ; H^{1}(\mathbb{R})\right)$ with $v(0)=u_{0}$ and that

$$
\left(1-\partial_{x}^{2}\right) u_{n_{k}} \rightarrow *\left(1-\partial_{x}^{2}\right) v \text { in } C_{w}([-T, T], \mathcal{M}) .
$$

In particular, $v_{t} \in L^{\infty}(]-T, T\left[; L^{2}(\mathbb{R})\right)$ and thus $v \in C\left([-T, T] ; L^{2}(\mathbb{R})\right)$. Since $v \in L^{\infty}(]-T, T\left[; H^{\frac{3}{2}-}(\mathbb{R})\right)$, this actually implies that $v \in C\left([-T, T] ; H^{\frac{3}{2}-}(\mathbb{R})\right)$ and, using again the equation, it follows that $v_{t} \in C\left(\mathbb{R} ; L^{2}(\mathbb{R})\right)$. Therefore, $v$ belongs to the uniqueness class which ensures that $v=u$ and thus the above weak convergence results hold for the whole sequence $\left\{u_{n}\right\}$. The conservation of $E(\cdot)$ together with these weak convergence results then lead to (2.4).

Let us now prove that $M(\cdot)$ is a conservation law for our solutions and that $y \in C_{t i}\left(\mathbb{R} ; \mathcal{M}_{+}\right)$. For this we apply the same arguments as above but for a smooth sequence $\left\{\tilde{u}_{0, n}\right\} \subset H^{3}(\mathbb{R}) \cap Y_{+}$that converges in the same sense to $u_{0} \in Y_{+}$. According to Proposition 2.1, $M(\cdot)$ is a conservation law for the solutions emanating from $\tilde{u}_{0, n}$ and, by hypothesis, $M\left(\tilde{u}_{0, n}\right) \rightarrow M\left(u_{0}\right)$. Therefore, the convergence result (2.8) ensures that $M(u(t)) \leq M\left(u_{0}\right)$ for all $t \neq 0$. But, approximating in the same way $u\left(t_{0}\right)$ for $t_{0} \neq 0$, the same arguments lead to $M(u(t)) \leq M\left(u\left(t_{0}\right)\right)$ for all $t \neq t_{0}$ which forces $M(\cdot)$ to be a conservation law. Hence, $M\left(\tilde{u}_{n}(t)\right) \rightarrow M(u(t))$ for all $t \in \mathbb{R}$. Since $\left\{\left(1-\partial_{x}^{2}\right) \tilde{u}_{n}(t)\right\} \subset \mathcal{M}_{+}$, it is well-known (see for instance Proposition 9 , page 61 , in [4]) that this convergence result together with the weak convergence (2.8) ensure the tight convergence of $\left(1-\partial_{x}^{2}\right) \tilde{u}_{n}(t)$ towards $y(t)$ for all $t \in \mathbb{R}$. Using again that $\left\{\partial_{t} \tilde{u}_{n}\right\}$ is bounded in $L^{\infty}(]-T, T\left[; L^{1}(\mathbb{R})\right)$ and Arzela-Ascoli's theorem, we obtain that $y \in C_{t i}\left(\mathbb{R} ; \mathcal{M}_{+}\right)$and that $(2.5)$ holds for $\left\{\tilde{u}_{n}\right\}$.

Now, coming back to the sequence $\left\{u_{n}\right\}$, we deduce from the tight convergence of $\left\{\left(1-\partial_{x}^{2}\right) u_{0, n}\right\}$ towards $u_{0}-u_{0, x x}$ together with the conservation of $M(\cdot)$ that $M\left(u_{n}(t)\right) \rightarrow M(u(t))$ for all $t \in \mathbb{R}$ and the same arguments as above lead to (2.5).

Finally (2.6) can be proven exactly in the same way, since $\left\{u_{0, n}\right\}$ is bounded in $Y_{+}$by Banach-Steinhaus theorem.

Remark 2.1. 3. of Proposition 2.2 ensures that (1.5) is a dynamical system in $Y_{+}$ endowed with its natural weak star topology, i.e.

$$
\varphi_{n} \rightarrow * \varphi \text { in } Y \text { iff } \varphi_{n} \rightarrow \varphi \text { in } H^{1}(\mathbb{R}) \text { and }\left(1-\partial_{x}^{2}\right) \varphi_{n} \rightarrow *\left(1-\partial_{x}^{2}\right) \varphi \text { in } \mathcal{M}(\mathbb{R}) \text {. }
$$

\section{Decay of $Y$-almost localized Solution moving to the Right}

Proposition 3.1. Let $u \in C\left(\mathbb{R} ; H^{1}\right)$ with $y=\left(1-\partial_{x}^{2}\right) u \in C_{w}(\mathbb{R} ; \mathcal{M}+)$ be a $Y$ almost localized solution of (1.5) with $\inf _{\mathbb{R}} \dot{x} \geq c_{0}>0$. Then there exists $C>0$ such that for all $t \in \mathbb{R}$, all $R>0$ and all $\Phi \in C(\mathbb{R})$ with $0 \leq \Phi \leq 1$ and $\operatorname{supp} \Phi \subset$ $[-R, R]^{c}$.

$$
\int_{\mathbb{R}}\left(u^{2}(t)+u_{x}^{2}(t)\right) \Phi(\cdot-x(t)) d x+c_{0}\langle\Phi(\cdot-x(t)), y(t)\rangle \leq C \exp (-R / 6) .
$$

To prove this proposition, the main tool is an almost monotonicity result for $E(u)+c_{0} M(u)$ at the right of an almost localized solution. Actually, the almost monotonicity is more general and says somehow that if $z(t)$ moves to the right with a positive speed strictly less that $\dot{x}(t)$ then the part of $E(u)+c_{0} M(u)$ at the right of $z(t)$ is almost decreasing as soon as $|z(t)-x(t)|$ stays large enough. 
As in [30], we introduce the $C^{\infty}$-function $\Psi$ defined on $\mathbb{R}$ by

$$
\Psi(x)=\frac{2}{\pi} \arctan (\exp (x / 6))
$$

It is easy to check that $\Psi(-\cdot)=1-\Psi$ on $\mathbb{R}, \Psi^{\prime}$ is a positive even function and that there exists $C>0$ such that $\forall x \leq 0$,

$$
|\Psi(x)|+\left|\Psi^{\prime}(x)\right| \leq C \exp (x / 6) .
$$

Moreover, by direct calculations, it is easy to check that

$$
\left|\Psi^{\prime \prime \prime}\right| \leq \frac{1}{2} \Psi^{\prime}
$$

Lemma 3.2. Let $0<\alpha<1$ and let $u \in C\left(\mathbb{R} ; H^{1}\right)$, with $y=\left(1-\partial_{x}^{2}\right) u \in$ $C_{w}\left(\mathbb{R} ; \mathcal{M}_{+}\right)$, be a solution of $(1.5)$ such that there exist $x: \mathbb{R} \rightarrow \mathbb{R}$ of class $C^{1}$ with $\inf _{\mathbb{R}} \dot{x} \geq c_{0}>0$ and $R_{0}>0$ with

$$
\|u(t)\|_{L^{\infty}\left(|x-x(t)|>R_{0}\right)} \leq \frac{(1-\alpha) c_{0}}{2^{6}}, \forall t \in \mathbb{R} .
$$

For $0<\beta \leq \alpha, 0 \leq \gamma \leq \frac{3}{2}(1-\alpha) c_{0}, R>0, t_{0} \in \mathbb{R}$ and any $C^{1}$-function

$$
z: \mathbb{R} \rightarrow \mathbb{R} \text { with }(1-\alpha) \dot{x}(t) \leq \dot{z}(t) \leq(1-\beta) \dot{x}(t), \quad \forall t \in \mathbb{R},
$$

setting

$$
I_{t_{0}}^{\mp R}(t)=\left\langle u^{2}(t)+u_{x}^{2}(t)+\gamma y(t), \Psi\left(\cdot-z_{t_{0}}^{\mp R}(t)\right)\right\rangle
$$

where

we have

$$
z_{t_{0}}^{\mp R}(t)=x\left(t_{0}\right) \mp R+z(t)-z\left(t_{0}\right)
$$

and

$$
I_{t_{0}}^{+R}\left(t_{0}\right)-I_{t_{0}}^{+R}(t) \leq K_{0} e^{-R / 6}, \quad \forall t \leq t_{0}
$$

for some constant $K_{0}>0$ that only depends on $E(u), c_{0}, R_{0}$ and $\beta$.

Proof. We first approximate $u\left(t_{0}\right)$ by the sequence of smooth functions $u_{0, n}=$ $\rho_{n} * u\left(t_{0}\right)$, with $\left\{\rho_{n}\right\}$ defined in $(2.3)$, that belongs to $H^{\infty}(\mathbb{R}) \cap Y_{+}$and converges ${ }^{4}$ to $u\left(t_{0}\right)$ in $Y$. According to Propositions 2.1 and 2.2 , the sequence of solutions $\left\{u_{n}\right\}$ to $(1.5)$ with $u_{n}\left(t_{0}\right)=u_{0, n}$ belongs to $C\left(\mathbb{R} ; H^{\infty}(\mathbb{R})\right)$ and for any fixed $T>0$ it holds

$$
\begin{aligned}
& u_{n} \quad \rightarrow \quad u \text { in } C\left(\left[t_{0}-T, t_{0}+T\right] ; H^{1}\right) \\
& y_{n} \rightarrow * \quad y \text { in } C_{t i}(] t_{0}-T, t_{0}+T[; \mathcal{M})
\end{aligned}
$$

where $y_{n}=u_{n}-\partial_{x}^{2} u_{n}$. In particular, for any fixed $T>0$, there exists $n_{0}=n_{0}(T) \geq$ 0 such that for any $n \geq n_{0}$,

$$
\left\|u_{n}-u\right\|_{L^{\infty}(] t_{0}-T, t_{0}+T[\times \mathbb{R})}<\frac{(1-\alpha) c_{0}}{2^{6}},
$$

which together with (3.5) forces

$$
\sup _{t \in] t_{0}-T, t_{0}+T[}\left\|u_{n}\right\|_{L^{\infty}\left(|x-x(t)|>R_{0}\right)}<\frac{(1-\alpha) c_{0}}{2^{5}} .
$$

\footnotetext{
${ }^{4} \mathrm{By}$ this me mean that $u_{0, n} \rightarrow u\left(t_{0}\right)$ in $H^{1}(\mathbb{R})$ and $\left(1-\partial_{x}^{2}\right) u_{0, n} \rightarrow\left(1-\partial_{x}^{2}\right) u\left(t_{0}\right)$ in $\mathcal{M}$.
} 
We first prove that (3.8) holds on $\left[t_{0}-T, t_{0}\right]$ with $u$ replaced by $u_{n}$ for $n \geq n_{0}$. The following computations hold for $u_{n}$ with $n \geq n_{0}$ but, to simplify the notation, we drop the index $n$. For any function $g \in C^{1}(\mathbb{R})$ it is not too hard to check that (see Appendix 7.1)

$$
\frac{d}{d t} \int_{\mathbb{R}}\left(u^{2}+u_{x}^{2}\right) g=\int_{\mathbb{R}} u u_{x}^{2} g^{\prime}+2 \int_{\mathbb{R}} u h g^{\prime}
$$

where $h:=\left(1-\partial_{x}^{2}\right)^{-1}\left(u^{2}+u_{x}^{2} / 2\right)$. Moreover, it easily follows from (1.4) that

$$
\begin{aligned}
\frac{d}{d t} \int_{\mathbb{R}} y g d x & =-\int_{\mathbb{R}} \partial_{x}(y u) g-\int_{\mathbb{R}} y u_{x} g \\
& =\int_{\mathbb{R}} y u g^{\prime}-\int_{\mathbb{R}}\left(u-u_{x x}\right) u_{x} g \\
& =\int_{\mathbb{R}} y u g^{\prime}+\frac{1}{2} \int_{\mathbb{R}}\left(u^{2}-u_{x}^{2}\right) g^{\prime}
\end{aligned}
$$

Applying (3.13)-(3.14) with $g(t, x)=\Psi\left(x-z_{t_{0}}^{R}(t)\right)$ we get

$$
\begin{aligned}
\frac{d}{d t} I_{t_{0}}^{+R}(t)= & -\dot{z}(t) \int_{\mathbb{R}} \Psi^{\prime}\left[u^{2}+u_{x}^{2}+\gamma y\right]+\frac{\gamma}{2} \int_{\mathbb{R}}\left(u^{2}-u_{x}^{2}\right) \Psi^{\prime} \\
& +\int_{\mathbb{R}}\left(u u_{x}^{2}+\gamma y u\right) \Psi^{\prime}+2 \int_{\mathbb{R}} u h \Psi^{\prime} \\
\leq & -\dot{z}(t) \int_{\mathbb{R}} \Psi^{\prime}\left[u^{2}+u_{x}^{2}+\gamma y\right]+\frac{\gamma}{2} \int_{\mathbb{R}}\left(u^{2}-u_{x}^{2}\right) \Psi^{\prime}+J_{1}+J_{2} .
\end{aligned}
$$

The crucial observation is that the second term in the right-hand side of (3.15) that provides from the momentum part of $I$ can be absorbed thanks to the term coming from the energy part of $I$. More precisely, for $0 \leq \gamma \leq \frac{3}{2}(1-\alpha) c_{0}$, it holds

$$
-\dot{z}(t) \int_{\mathbb{R}} \Psi^{\prime}\left[u^{2}+u_{x}^{2}+\gamma y\right]+\frac{\gamma}{2} \int_{\mathbb{R}}\left(u^{2}-u_{x}^{2}\right) \Psi^{\prime} \leq-\frac{(1-\alpha) c_{0}}{4} \int_{\mathbb{R}} \Psi^{\prime}\left[u^{2}+u_{x}^{2}+\gamma y\right]
$$

Now, the terms $J_{1}$ and $J_{2}$ are treated as usually. To estimate $J_{1}$ we divide $\mathbb{R}$ into two regions relating to the size of $|u|$ as follows

$$
\begin{aligned}
J_{1}(t) & =\int_{|x-x(t)|<R_{0}}\left(u u_{x}^{2}+\gamma y u\right) \Psi^{\prime}+\int_{|x-x(t)|>R_{0}}\left(u u_{x}^{2}+\gamma y u\right) \Psi^{\prime} \\
& =J_{11}+J_{12} .
\end{aligned}
$$

Observe that (3.6) ensures that $\dot{x}(t)-\dot{z}(t) \geq \beta c_{0}$ for all $t \in \mathbb{R}$ and thus, for $|x-x(t)|<R_{0}$,

$$
x-z_{t_{0}}^{R}(t)=x-x(t)-R+(x(t)-z(t))-\left(x\left(t_{0}\right)-z\left(t_{0}\right)\right) \leq R_{0}-R-\beta c_{0}\left(t_{0}-t\right)
$$

and thus the decay properties of $\Psi^{\prime}$ ensure that

$$
\begin{aligned}
J_{11}(t) & \lesssim\left[\|u(t)\|_{L^{\infty}}\left(\left\|u_{x}(t)\right\|_{L^{2}}^{2}+c_{0}\|y(t)\|_{L^{1}}\right)\right] e^{R_{0} / 6} e^{-R / 6} e^{-\frac{\beta}{6} c_{0}\left(t_{0}-t\right)} \\
& \lesssim\left\|u_{0}\right\|_{H^{1}}\left(\left\|u_{0}\right\|_{H^{1}}^{2}+c_{0}\left\|y_{0}\right\|_{L^{1}}\right) e^{R_{0} / 6} e^{-R / 6} e^{-\frac{\beta}{6} c_{0}\left(t_{0}-t\right)}
\end{aligned}
$$

On the other hand, (3.12) ensures that for all $t \in\left[t_{0}-T, t_{0}\right]$ it holds

$$
\begin{aligned}
J_{12} & \leq 4\|u\|_{L^{\infty}\left(|x-x(t)|>R_{0}\right)} \int_{|x-x(t)|>R_{0}}\left(u_{x}^{2}+\gamma y\right) \Psi^{\prime} \\
& \leq \frac{(1-\alpha) c_{0}}{8} \int_{|x-x(t)|>R_{0}}\left(u_{x}^{2}+\gamma y\right) \Psi^{\prime} .
\end{aligned}
$$


It thus remains to estimate $J_{2}(t)$. For this, we decompose again $\mathbb{R}$ into two regions relating to the size of $|u|$. First proceeding as in (3.18) we easily check that

$$
\begin{aligned}
& \int_{|x-x(t)|<R_{0}} u \Psi^{\prime}\left(1-\partial_{x}^{2}\right)^{-1}\left(2 u^{2}+u_{x}^{2}\right) \\
& \leq 4\|u\|_{L^{\infty}} \sup _{|x-x(t)|<R_{0}}\left|\Psi^{\prime}\left(x-z_{t_{0}}^{R}(t)\right)\right| \int_{\mathbb{R}} e^{-|x|} *\left(u^{2}+u_{x}^{2}\right) d x \\
& \leq C\left\|u_{0}\right\|_{H^{1}}^{3} e^{R_{0} / 6} e^{-R / 6} e^{-\frac{\beta}{6} c_{0}\left(t-t_{0}\right)}
\end{aligned}
$$

since

$$
\forall f \in L^{1}(\mathbb{R}), \quad\left(1-\partial_{x}^{2}\right)^{-1} f=\frac{1}{2} e^{-|x|} * f \quad .
$$

Now in the region $|x-x(t)|>R_{0}$, noticing that $\Psi^{\prime}$ and $u^{2}+u_{x}^{2} / 2$ are non-negative, we get

$$
\begin{aligned}
& \int_{|x-x(t)|>R_{0}} u \Psi^{\prime}\left(1-\partial_{x}^{2}\right)^{-1}\left(2 u^{2}+u_{x}^{2}\right) \\
\leq & \|u(t)\|_{L^{\infty}\left(|x-x(t)|>R_{0}\right)} \int_{|x-x(t)|>R_{0}} \Psi^{\prime}\left(\left(1-\partial_{x}^{2}\right)^{-1}\left(2 u^{2}+u_{x}^{2}\right)\right. \\
\leq & \|u(t)\|_{L^{\infty}\left(|x-x(t)|>R_{0}\right)} \int_{\mathbb{R}}\left(2 u^{2}+u_{x}^{2}\right)\left(1-\partial_{x}^{2}\right)^{-1} \Psi^{\prime}
\end{aligned}
$$

On the other hand, from (3.4) and (3.21) we infer that

$$
\left(1-\partial_{x}^{2}\right) \Psi^{\prime} \geq \frac{1}{2} \Psi^{\prime} \Rightarrow\left(1-\partial_{x}^{2}\right)^{-1} \Psi^{\prime} \leq 2 \Psi^{\prime} .
$$

Therefore, on account of (3.12),

$$
\begin{aligned}
& \int_{|x-x(t)|>R_{0}} u \Psi^{\prime}\left(1-\partial_{x}^{2}\right)^{-1}\left(2 u^{2}+u_{x}^{2}\right) \\
& \leq 2\|u(t)\|_{L^{\infty}\left(|x-x(t)|>R_{0}\right)} \int_{\mathbb{R}}\left(2 u^{2}+u_{x}^{2}\right) \Psi^{\prime} \\
& \leq \frac{(1-\alpha) c_{0}}{8} \int_{\mathbb{R}}\left(u^{2}+u_{x}^{2}\right) \Psi^{\prime}
\end{aligned}
$$

Gathering (3.16), (3.18), (3.19), (3.20) and (3.23) we conclude that there exists $C$ only depending on $R_{0}$ and $E(u)$ such that for $R \geq R_{0}$ and $t \in\left[-T+t_{0}, t_{0}\right]$ it holds

$$
\frac{d}{d t} I_{t_{0}}^{+R}(t) \leq C e^{-R / 6} e^{-\frac{\beta}{6}\left(t_{0}-t\right)} .
$$

Integrating between $t$ and $t_{0}$ we obtain (3.8) for any $t \in\left[t_{0}-T, t_{0}\right]$ and $u$ replaced by $u_{n}$ with $n \geq n_{0}$. Note that the constant appearing in front of the exponential now also depends on $\beta$. The convergence results (3.10)-(3.11) then ensure that (3.8) holds also for $u$ and $t \in\left[t_{0}-T, t_{0}\right]$ and the result for $t \leq t_{0}$ follows since $T>0$ is arbitrary. Finally, (3.9) can be proven in exactly the same way by noticing that for $|x-x(t)|<R_{0}$ it holds

$$
x-z_{t_{0}}^{-R}(t)=x-x(t)+R+(x(t)-z(t))-\left(x\left(t_{0}\right)-z\left(t_{0}\right)\right) \geq-R_{0}+R+\beta c_{0}\left(t-t_{0}\right) .
$$


Proof of Proposition 3.1 First, since $u$ is $Y$-almost localized, it is clear that $u$ satisfies the hypotheses of Lemma 3.2 for $\alpha=1 / 3$. We fix $\alpha=1 / 3$ and take $\beta=1 / 3, \gamma=c_{0}$ and $z(\cdot)=\frac{2}{3} x(\cdot)$ which clearly satisfy (3.6). Let us show that $I_{t_{0}}^{+R}(t) \underset{t \rightarrow-\infty}{\longrightarrow} 0$ which together with (3.8) will clearly lead to

$$
I_{t_{0}}^{+R}\left(t_{0}\right) \leq C e^{-R / 6}
$$

For $R_{\varepsilon}>0$ to be specified later we decompose $I_{t_{0}}^{+R}$ into

$$
\begin{aligned}
I_{t_{0}}^{+R}(t)= & \left\langle u^{2}(t)+u_{x}^{2}(t)+c_{0} y(t), \Psi\left(\cdot-z_{t_{0}}^{R}(t)\right)\left(1-\phi\left(\frac{-x(t)}{R_{\varepsilon}}\right)\right)\right\rangle \\
& +\left\langle u^{2}(t)+u_{x}^{2}(t)+c_{0} y(t), \Psi\left(\cdot-z_{t_{0}}^{R}(t)\right) \phi\left(\frac{-x(t)}{R_{\varepsilon}}\right)\right\rangle \\
= & I_{1}(t)+I_{2}(t) .
\end{aligned}
$$

where $\phi \in C^{\infty}(\mathbb{R})$ is supported in $[-1,1]$ with $0 \leq \phi \leq 1$ on $[-1,1]$ and $\phi \equiv 1$ on $[-1 / 2,1 / 2]$. From the $Y$-almost localization hypothesis, for any $\varepsilon>0$ there exists $R_{\varepsilon}>0$ such that $I_{1}(t) \leq \varepsilon / 2$. On the other hand, we observe that

$$
I_{2}(t) \leq\left(\left\|u_{0}\right\|_{H^{1}}^{2}+c_{0}\left\|y_{0}\right\|_{\mathcal{M}}\right) \Psi\left(R_{\varepsilon}-R-\frac{1}{3}\left(x\left(t_{0}\right)-x(t)\right)\right) .
$$

But $\dot{x}>c_{0}>0$ obviously imply that, for $|x-x(t)| \leq R_{\varepsilon}$,

$x-z_{t_{0}}^{+R}(t)=x-x(t)-R-\frac{1}{3}\left(x\left(t_{0}\right)-x(t)\right) \leq R_{\varepsilon}-R-\frac{1}{3} c_{0}\left(t_{0}-t\right) \underset{t \rightarrow-\infty}{\longrightarrow}-\infty$

which proves our claim since $\lim _{x \rightarrow-\infty} \Psi(x)=0$.

It follows from (3.26) that for all $t \in \mathbb{R}$, all $x_{0}>0$ and all $\Phi \in C(\mathbb{R})$ with $0 \leq \Phi \leq 1$ and $\operatorname{supp} \Phi \subset\left[x_{0},+\infty[\right.$.

$$
\int_{\mathbb{R}}\left(u^{2}(t)+u_{x}^{2}(t)\right) \Phi(\cdot-x(t)) d x+c_{0}\langle\Phi(\cdot-x(t)), y(t)\rangle \leq C \exp (-R / 6) .
$$

The invariance of $(\mathrm{C}-\mathrm{H})$ under the transformation $(t, x) \mapsto(-t,-x)$ yields the result for $\left.\operatorname{supp} \Phi \subset]-\infty,-x_{0}\right]$ which completes the proof of the proposition.

\section{LiOUVILle RESUlt FOR $Y$-ALMOST LOCALIZED SOLUTION MOVING TO THE RIGHT}

In this section we will need the following lemma (see for instance [24])

Lemma 4.1. Let $\mu$ be a finite nonnegative measure on $\mathbb{R}$. Then $\mu$ is the sum of a nonnegative non atomic measure $\nu$ and a countable sum of positive Dirac measures (the discrete part of $\mu$ ). Moreover, for all $\varepsilon>0$ there exists $\delta>0$ such that, if $I$ is an interval of length less than $\delta$, then $\nu(I) \leq \varepsilon$.

\subsection{Boundedness from above of the support of the momentum density.}

Proposition 4.2. Let $u \in C\left(\mathbb{R} ; Y_{+}\right)$be a $Y$-almost localized solution of (1.5) with $\dot{x} \geq c_{0}>0$. There exists $r_{0}>0$ such that for all $t \in \mathbb{R}$, it holds

$$
\left.\operatorname{supp} y(t, \cdot+x(t)) \subset]-\infty, r_{0}\right]
$$

and

$$
u\left(t, x(t)+r_{0}\right)=-u_{x}\left(t, x(t)+r_{0}\right) \geq \frac{e^{-2 r_{0}}}{4 \sqrt{r_{0}}} \sqrt{E(u)} .
$$


Proof. Clearly, it suffices to prove the result for $t=0$. Let $u \in C\left(\mathbb{R} ; H^{1}\right)$, with $y=$ $u-u_{x x} \in C_{w}\left(\mathbb{R} ; \mathcal{M}_{+}\right)$, be a $Y$-almost localized solution to (1.5) and let $\phi \in C^{\infty}(\mathbb{R})$ with $\phi \equiv 0$ on $\mathbb{R}_{-}, \phi^{\prime} \geq 0$ and $\phi \equiv 1$ on $[1,+\infty]$. We claim that there exists $r_{0}>0$ such that

$$
\left\langle y(0), \phi\left(\cdot-\left(x(0)+r_{0}\right)\right)\right\rangle=0
$$

which proves the result since $y(0) \in \mathcal{M}_{+}$.

We prove (4.3) by contradiction. We approximate $u_{0}=u(0)$ by the sequence of smooth functions $u_{0, n}=\rho_{n} * u_{0}$ that belongs to $H^{\infty}(\mathbb{R}) \cap Y_{+}$so that (2.4)-(2.5) hold for any $T>0$. We denote by $u_{n}$ the solution to (1.5) emanating from $u_{0, n}$ and by $y_{n}=u_{n}-u_{n, x x}$ its momentum density. Let us recall that Proposition 2.1 ensures that $u_{n} \in C\left(\mathbb{R} ; H^{\infty}(\mathbb{R})\right)$ and $y_{n} \in C_{w}\left(\left(\mathbb{R} ; L^{1}(\mathbb{R})\right)\right.$. We fix $T>0$ and we take $n_{0} \in \mathbb{N}$ large enough so that for all $n \geq n_{0}$,

$$
\left\|u_{n}-u\right\|_{L^{\infty}(]-T, T\left[; H^{1}\right)}<\frac{1}{10} \min \left(c_{0},\|u(0)\|_{H^{1}}\right)
$$

and

$$
\left\|y_{0, n}-y_{0}\right\|_{\mathcal{M}}<\frac{\varepsilon_{0}}{2}
$$

where $\varepsilon_{0}>0$ will be specified later. Thanks to the $Y$-almost localization of $u$, there exists $r_{0}>0$ such that

$$
\|u(t)\|_{H^{1}(\mathbb{R} /] x(t)-r_{0}, x(t)+r_{0}[)} \leq \frac{1}{10} \min \left(c_{0},\|u(0)\|_{H^{1}}\right), \forall t \in \mathbb{R} .
$$

Note that by Sobolev's embedding theorem, it also holds

$$
u(t, x(t)+x) \leq \frac{1}{10} \min \left(c_{0},\|u(0)\|_{H^{1}}\right), \forall(|x|, t) \in\left[r_{0},+\infty[\times \mathbb{R} .\right.
$$

Combining these two estimates with (4.4) we infer that for $n \geq n_{0}$,

$$
\left\|u_{n}(t)\right\|_{H^{1}(\mathbb{R} /] x(t)-r_{0}, x(t)+r_{0}[)} \leq \frac{1}{5} \min \left(c_{0},\|u(0)\|_{H^{1}}\right), \forall t \in[-T, T]
$$

and

$$
u_{n}(t, x(t)+x) \leq \frac{1}{5} \min \left(c_{0},\|u(0)\|_{H^{1}}\right), \forall(|x|, t) \in\left[r_{0},+\infty[\times[-T, T] .\right.
$$

Now, we introduce the flow $q_{n}$ associated with $u_{n}$ defined by

$$
\left\{\begin{array}{rlrl}
q_{n, t}(t, x) & =u_{n}\left(t, q_{n}(t, x)\right) & & ,(t, x) \in \mathbb{R}^{2} \\
q_{n}(0, x) & =x & & x \in \mathbb{R}
\end{array} .\right.
$$

Following [11], we know that for any $t \in \mathbb{R}$,

$$
y_{n}(0, x)=y_{n}\left(t, q_{n}(t, x)\right) q_{n, x}(t, x)^{2}
$$

Indeed, on one hand, (1.4) clearly ensures that

$$
\frac{\partial}{\partial t}\left(y_{n}\left(t, q_{n}(t, x)\right) e^{2 \int_{0}^{t} u_{n, x}\left(s, q_{n}(s, x)\right) d s}\right)=0
$$

and, on the other hand, (4.10) ensures that $q_{n, x}(0, x)=1, \forall x \in \mathbb{R}$, and

$$
\frac{\partial}{\partial t} q_{n, x}(t, x)=u_{x}(t, q(t, x)) q_{x}(t, x) \Rightarrow q_{n, x}(t, x)=\exp \left(\int_{0}^{t} u_{x}(s, q(s, x)) d s\right) .
$$


We claim that for all $n \geq n_{0}$ and $t \in[-T, 0]$,

$$
q_{n}\left(t, x(0)+r_{0}\right)-x(t) \geq r_{0}+\frac{c_{0}}{2}|t| .
$$

Indeed, fixing $n \geq n_{0}$, in view of (4.9) and the continuity of $u_{n}$ there exists $t_{0} \in$ $\left[-T, 0\left[\right.\right.$ such that for all $t \in\left[t_{0}, 0\right]$,

$$
u_{n}\left(t, q_{n}\left(t, x(0)+r_{0}\right)\right) \leq \frac{c_{0}}{4}
$$

and thus according to (4.10), for all $t \in\left[t_{0}, 0\right]$,

which leads to

$$
\frac{d}{d t} q_{n}\left(t, x(0)+r_{0}\right) \leq \frac{c_{0}}{4}
$$

$$
q_{n}\left(t, x(0)+r_{0}\right)-x(t) \geq r_{0}+\frac{c_{0}}{2}|t|, \quad t \in\left[t_{0}, 0\right] .
$$

This proves (4.13) by a continuity argument. We thus deduce from Proposition 3.1 that for all $t \in[-T, 0]$ and all $x \geq 0$,

$$
u\left(t, q_{n}\left(t, x(0)+r_{0}+x\right) \leq C \exp \left(-\frac{1}{6}\left(r_{0}+c_{0}|t| / 2\right)\right)\right.
$$

Therefore, in view of (2.4) and (2.2), there exists $n_{1} \geq n_{0}$ such that for all $t \in[-T, 0]$ and all $x \geq 0$,

$u_{n}\left(t, q_{n}\left(t, x(0)+r_{0}+x\right)+\mid u_{n, x}\left(t, q_{n}\left(t, x(0)+r_{0}+x\right) \mid \leq 4 C \exp \left(-\frac{1}{6}\left(r_{0}+c_{0}|t| / 2\right)\right)\right.\right.$

The formula (see (4.12))

$$
q_{n, x}(t, x)=\exp \left(-\int_{t}^{0} u_{n, x}\left(s, q_{n}(s, x)\right) d s\right)
$$

thus ensures that $\forall t \in[-T, 0], \forall x \geq 0$ and $\forall n \geq n_{0}$,

$\exp \left(-4 C \int_{-T}^{0} e^{-\frac{1}{6}\left(r_{0}+c_{0}|s| / 2\right)} d s\right) \leq q_{n, x}\left(t, x(0)+r_{0}+x\right) \leq \exp \left(4 C \int_{-T}^{0} e^{-\frac{1}{6}\left(r_{0}+c_{0}|s| / 2\right)} d s\right)$

Setting $C_{0}:=e^{\frac{48 C e^{-r_{0} / 6}}{c_{0}}}$ this leads to

$$
\frac{1}{C_{0}} \leq q_{n, x}\left(t, x(0)+r_{0}+x\right) \leq C_{0}, \forall t \in[-T, 0] .
$$

Now, if (4.3) would not be true then, in view of (4.5) there exists $r_{0}^{\prime}>r_{0}$ and $\varepsilon_{0}>0$ such that $\forall n \geq n_{1}$,

$$
\int_{x(0)+r_{0}}^{x(0)+r_{0}^{\prime}} y_{n}(0, x) d x \geq \varepsilon_{0}>0 .
$$

It then follows from (4.11) that for all $t \in[-T, 0]$,

$$
\int_{x(0)+r_{0}}^{x(0)+r_{0}^{\prime}} y_{n}\left(t, q_{n}(t, x)\right) q_{n, x}(t, x)^{2} d x \geq \varepsilon_{0}
$$

and (4.17) leads to

$$
\int_{x(0)+r_{0}}^{x(0)+r_{0}^{\prime}} y_{n}\left(t, q_{n}(t, x)\right) q_{n, x}(t, x) d x \geq C_{0}^{-1} \varepsilon_{0}
$$


Therefore, the change of variables $z=q_{n}(t, x)$ yields

$$
\int_{q_{n}\left(t, x(0)+r_{0}\right)}^{q_{n}\left(t, x(0)+r_{0}^{\prime}\right)} y_{n}(t, z) d z \geq C_{0}^{-1} \varepsilon_{0}
$$

and (4.13) ensures that

$$
\int_{x(t)+r_{0}+c_{0}|t| / 2}^{+\infty} y_{n}(t, z) d z \geq C_{0}^{-1} \varepsilon_{0}, \forall t \in[-T, 0] .
$$

Letting $n \rightarrow+\infty$ using (2.5) and then letting $T \rightarrow \infty$, this ensures that

$$
\left\langle y(t), \phi\left(\cdot-x(t)-r_{0}-c_{0}|t| / 2\right)\right\rangle \geq C_{0}^{-1} \varepsilon_{0}, \forall t \leq 0 .
$$

This clearly contradicts the $Y$-almost localization of $u$ and thus completes the proof of (4.1).

Let us now prove (4.2). We first notice that thanks to (4.6) and the conservation of the $H^{1}$-norm it holds

$$
\|u(t, \cdot-x(t))\|_{H^{1}(]-r_{0}, r_{0}[)} \geq \frac{1}{2} \sqrt{E(u)} .
$$

But since for all $t \in \mathbb{R},\left|u_{x}(t)\right| \leq u(t)$ on $\mathbb{R}$, this forces

$$
\max _{\left[-r_{0}, r_{0}\right]} u^{2}(t, \cdot-x(t)) \geq \frac{1}{2 r_{0}}\|u(t, \cdot-x(t))\|_{L^{2}(]-r_{0}, r_{0}[)}^{2} \geq \frac{1}{8 r_{0}} E(u) .
$$

Moreover, since $u_{x} \geq-u$ on $\mathbb{R}^{2}$, for any $\left(t, x_{0}\right) \in \mathbb{R}^{2}$ it holds

$$
u(t, x) \leq u\left(t, x_{0}\right) e^{-x+x_{0}}, \quad \forall x \leq x_{0} .
$$

Applying this estimate with $x_{0}=x(t)+r_{0}$ we obtain that

$$
u\left(t, x(t)+r_{0}\right) \geq \max _{\left[-r_{0}, r_{0}\right]} u(t, \cdot-x(t)) e^{-2 r_{0}}
$$

which, combined with (4.19) yields (4.2).

\subsection{Study of the first jump of $u_{x}$. We define}

$$
\left.\left.x_{+}(t)=\inf \{x \in \mathbb{R}, \operatorname{supp} y(t) \subset]-\infty, x(t)+x\right]\right\}
$$

In the sequel we set

$$
\alpha_{0}:=\frac{e^{-2 r_{0}}}{4 \sqrt{r_{0}}} \sqrt{E(u)}
$$

to simplify the expressions. According to Proposition $4.2, t \mapsto x_{+}(t)$ is well defined with values in $\left.]-\infty, r_{0}\right]$ and

$$
u\left(t, x(t)+x_{+}(t)\right)=-u_{x}\left(t, x(t)+x_{+}(t)\right) \geq \alpha_{0} .
$$

The following lemma ensures that $t \mapsto x(t)+x_{+}(t)$ is an integral line of $u$.

Lemma 4.3. For all $t \in \mathbb{R}$, it holds

$$
x(t)+x_{+}(t)=q\left(t, x(0)+x_{+}(0)\right) .
$$

where $q(\cdot, \cdot)$ is defined by

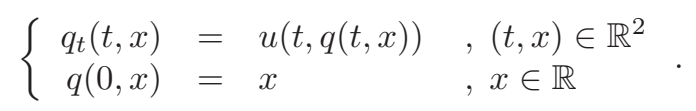


Proof. First, it is worth noticing that the result would follow directly from the definition of $x_{+}(\cdot)$ and $q(\cdot)$ if $u$ would belong to $C\left(\mathbb{R} ; H^{3}(\mathbb{R})\right)$. Since $(1.5)$ is reversible with time, it suffices to prove that (4.21) holds for all $t \in[0,1]$. We proceed by contradiction by assuming that there exits $\left.\left.t_{0} \in\right] 0,1\right]$ such that $q\left(t_{0}, x(0)+x_{+}(0)=\right.$ $x\left(t_{0}\right)+x_{+}\left(t_{0}\right)+\lambda_{0}$ with $\lambda_{0} \neq 0$. We separate the cases $\lambda_{0}>0$ and $\lambda_{0}<0$.

In the case $\lambda_{0}<0$, by the continuity and monotonicity of $x \mapsto q(t, x), t \in \mathbb{R}$, there exists $\delta_{0}>0$ such that $q\left(t_{0}, x(0)+x_{+}(0)+\delta_{0}\right)<x\left(t_{0}\right)+x_{+}\left(t_{0}\right)-\frac{\left|\lambda_{0}\right|}{2}$. We approximate $u$ at time $t=0$ by the sequence $\varphi_{n}=\rho_{n} * u(0)$ where $\rho_{n}$ is defined in (2.3). By construction $\varphi_{n} \in H^{\infty}(\mathbb{R}) \cap Y_{+}$and $\left(1-\partial_{x}^{2}\right) \varphi_{n} \equiv 0$ on $\left[x(0)+x_{+}(0)+\delta_{0},+\infty\left[\right.\right.$ as soon as $n>2 / \delta_{0}$. Let $u_{n}$ be the solution of (1.5) such that $u_{n}\left(t_{0}\right)=\varphi_{n}$. Defining $q_{n}: \mathbb{R} \rightarrow \mathbb{R}$ by

$$
\left\{\begin{array}{rl}
\frac{d}{d t} q_{n}(t) & =u_{n}\left(t, q_{n}(t)\right) \\
q_{n}(0) & =x\left(t_{0}\right)+x_{+}\left(t_{0}\right)+\delta_{0}
\end{array} \quad, \forall t \in \mathbb{R}\right.
$$

it follows from $(4.11)$ that $\left(1-\partial_{x}^{2}\right) u_{n}(0, \cdot) \equiv 0$ on $\left[q_{n}\left(t_{0}\right),+\infty\left[\right.\right.$ for $n>2 / \delta_{0}$. On account of $(2.4), q_{n}(\cdot) \rightarrow q\left(\cdot, x\left(t_{0}\right)+x_{+}\left(t_{0}\right)+\delta_{0}\right)$ in $C([0,1])$ and thus $(1-$ $\left.\partial_{x}^{2}\right) u_{n}\left(t_{0}\right) \equiv 0$ on $\left[x\left(t_{0}\right)+x_{+}\left(t_{0}\right)-\frac{\left|\lambda_{0}\right|}{4},+\infty[\right.$ for $n$ large enough. (2.5) then ensures that $\left.\operatorname{supp} y\left(t_{0}\right) \subset\right]-\infty, x\left(t_{0}\right)+x_{+}\left(t_{0}\right)-\frac{\left|\lambda_{0}\right|}{8}[$ which contradicts the definiton of $x_{+}\left(t_{0}\right)$.

In the case $\lambda_{0}>0$, there exists $\delta_{0}>0$ such that $q\left(t_{0}, x(0)+x_{+}(0)-\delta_{0}\right)>$ $x\left(t_{0}\right)+x_{+}\left(t_{0}\right)+\frac{\left|\lambda_{0}\right|}{2}$. Then we obtain again a contradiction by following the same arguments, exchanging the role of $t=0$ and $t=t_{0}$. This completes the proof of the lemma.

In the sequel we define $q^{*}: \mathbb{R} \rightarrow \mathbb{R}$ by

$$
q^{*}(t)=q\left(t, x(0)+x_{+}(0)\right)=x(t)+x_{+}(t), \quad \forall t \in \mathbb{R} .
$$

Proposition 4.4. Let $a: \mathbb{R} \rightarrow \mathbb{R}$ be the function defined by

$$
a(t)=u_{x}\left(t, q^{*}(t)-\right)-u_{x}\left(t, q^{*}(t)+\right), \quad \forall t \in \mathbb{R} .
$$

Then $a(\cdot)$ is a bounded non decreasing differentiable function on $\mathbb{R}$ with values in $\left[\frac{\alpha_{0}}{8}, 2 \sqrt{E(u)}\right]$ such that

$$
a^{\prime}(t)=\frac{1}{2}\left(u^{2}-u_{x}^{2}\right)\left(t, q^{*}(t)-\right), \forall t \in \mathbb{R} .
$$

Proof. First, the fact that $a(t) \leq 2 \sqrt{E(u)}$ follows directly from $\left|u_{x}\right| \leq u \leq\|u\|_{H^{1}}$. To prove that $a(t) \geq \frac{\alpha_{0}}{8}$, we proceed by contradiction. So let us assume that there exists $t_{0} \in \mathbb{R}$ such that $a\left(t_{0}\right)<\alpha_{0} / 8$. Since $y\left(t_{0}\right) \in \mathcal{M}_{+}$with $\operatorname{supp} y\left(t_{0}\right) \subset$ ] $\left.-\infty, q^{*}\left(t_{0}\right)\right]$, according to Lemma 4.1 we must have

$$
\lim _{z \nearrow_{q^{*}\left(t_{0}\right)}}\left\|y\left(t_{0}\right)\right\|_{\mathcal{M}(] z,+\infty[)}<\frac{\alpha_{0}}{8} .
$$

Without loss of generality we can assume that $t_{0}=0$ and thus there exists $\beta_{0}>0$ such that

$$
\|y(0)\|_{\left.\mathcal{M}(] q^{*}(0)\right)-\beta_{0},+\infty[)}<\frac{\alpha_{0}}{8} .
$$

By convoluting $u_{0}$ by $\rho_{n}$ (see (2.3)), for some $n \geq 0$, we can approach $u_{0}$ by a smooth function $\tilde{u}_{0} \in Y_{+} \cap H^{\infty}(\mathbb{R})$. Taking $n$ large enough, we may assume that there exists $\tilde{x}_{+}>x_{+}(0)$ close to $x_{+}(0)$, such that

$$
\tilde{y}_{0}=\left(1-\partial_{x}^{2}\right) \tilde{u}_{0} \equiv 0 \text { on }\left[x(0)+\tilde{x}_{+},+\infty[\right.
$$


and

$$
\left\|\tilde{y}_{0}\right\|_{L^{1}(] x(0)+\tilde{x}_{+}-\beta_{0},+\infty[)} \leq \frac{\alpha_{0}}{8}+\frac{\alpha_{0}}{2^{6}},
$$

where $\tilde{y}_{0}=\tilde{u}_{0}-\tilde{u}_{0, x x}$. Moreover, defining $\tilde{q}_{2}: \mathbb{R} \rightarrow \mathbb{R}$ by

$$
\tilde{q}_{2}(t)=\tilde{q}\left(t, x(0)+\tilde{x}_{+}\right)
$$

where $\tilde{q}(\cdot, \cdot)$ is defined by $(4.22)$ with $u$ replaced by $\tilde{u},(2.4)$ enables us to assume that the emanating solution $\tilde{u}$ satisfies

$$
\|u(t)-\tilde{u}(t)\|_{H^{1}} \leq \frac{\alpha_{0}}{2^{6}}
$$

and

$$
\left|q^{*}(t)-\tilde{q}_{2}(t)\right|<\frac{\alpha_{0}}{2^{6} \sqrt{E(u)}}
$$

for all $t \in\left[-t_{1}, t_{1}\right]$ with $t_{1}>0$ to specified later. It is worth noticing that (4.29)(4.30), (4.27), (4.11), (4.2) and the mean-value theorem - recall that $\left|u_{x}\right| \leq u \leq$ $\sqrt{E(u)}$ - then ensure that

$$
-\tilde{u}_{x}\left(t, \tilde{q}_{2}(t)\right)=\tilde{u}\left(t, \tilde{q}_{2}(t)\right) \geq\left(1-2^{-5}\right) \alpha_{0} \quad \forall t \in\left[-t_{1}, t_{1}\right] .
$$

We claim that for all $t \in\left[-t_{1}, 0\right]$ it holds

$$
\tilde{u}_{x}(t, x) \leq-\frac{3 \alpha_{0}}{4} \quad \text { on } \quad\left[\tilde{q}_{1}(t), \tilde{q}_{2}(t)\right],
$$

where $\tilde{q}_{1}(t)$ is defined by $\tilde{q}_{1}(t)=\tilde{q}\left(t, x(0)+\tilde{x}_{+}-\beta_{0}\right)$.

To see this, for $\gamma>0$, we set

$$
A_{\gamma}=\left\{t \in \mathbb{R}_{-} / \forall \tau \in[t, 0], u_{x}(\tau, x)<-\gamma \text { on }\left[\tilde{q}_{1}(\tau), \tilde{q}_{2}(\tau)\right]\right\} .
$$

Recalling (4.2), (4.28), (4.31) and that $\tilde{u} \geq 0$, we get for $0 \leq \beta \leq \beta_{0}$,

$$
\begin{aligned}
\tilde{u}_{x}\left(0, x(0)+\tilde{x}_{+}-\beta\right) & \leq \tilde{u}_{x}\left(0, x(0)+\tilde{x}_{+}\right)+\left\|\tilde{y}_{0}\right\|_{L^{1}(] x(0)+\tilde{x}_{+}-\beta_{0},+\infty[)} \\
& \leq-\alpha_{0}+\frac{\alpha_{0}}{2^{5}}+\frac{\alpha_{0}}{8}+\frac{\alpha_{0}}{2^{5}}<-\frac{3 \alpha_{0}}{4},
\end{aligned}
$$

which ensures that $A_{\underline{3 \alpha_{0}}}$ is non empty. By a continuity argument, it thus suffices to prove that $A_{\frac{\alpha_{0}}{2}} \subset{\stackrel{4}{4} \frac{3 \alpha_{0}}{4}}$. First we notice that for any $t \in A_{\frac{\alpha_{0}}{2}}$ and any $x \in$ $\left[\tilde{q}_{1}(t), \tilde{q}_{2}(t)\right]$, the definition of $A_{\frac{\alpha_{0}}{2}}$ ensures that

$$
\tilde{q}_{x}(t, x)=\exp \left(-\int_{t}^{0} \tilde{u}_{x}(\tau, \tilde{q}(\tau, x)) d \tau\right) \geq 1,
$$

where $\tilde{q}(\cdot, \cdot)$ is the flow associated to $\tilde{u}$ by $(4.22)$. Therefore, $\tilde{u} \geq 0, \tilde{y} \geq 0$, a change of variables, (4.11) and (4.28) ensure that for any $x \in\left[\tilde{q}_{1}(t), \tilde{q}_{2}(t)\right]$,

$$
\begin{gathered}
\int_{x}^{\tilde{q}_{2}(t)} \tilde{u}_{x x}(t, s) d s \geq-\int_{x}^{\tilde{q}_{2}(t)} \tilde{y}(t, s) d s \geq-\int_{\tilde{q}_{1}(t)}^{\tilde{q}_{2}(t)} \tilde{y}(t, s) d s=-\int_{x(0)+\tilde{x}_{+}-\beta_{0}}^{x(0)+\tilde{x}_{+}} \tilde{y}(t, \tilde{q}(t, s)) \tilde{q}_{x}(t, s) d s \\
\geq-\int_{x(0)+\tilde{x}_{+}-\beta_{0}}^{x(0)+\tilde{x}_{+}} \tilde{y}(t, \tilde{q}(t, s)) \tilde{q}_{x}(t, s)^{2} d s=-\int_{x(0)+\tilde{x}_{+}-\beta_{0}}^{x(0)+\tilde{x}_{+}} \tilde{y}_{0}(s) d s \geq-\frac{\alpha_{0}}{8}-\frac{\alpha_{0}}{2^{6}}
\end{gathered}
$$

and (4.31) yields

$$
\tilde{u}_{x}(t, x)=\tilde{u}_{x}\left(t, \tilde{q}_{2}(t)\right)-\int_{x}^{\tilde{q}_{2}(t)} \tilde{u}_{x x}(t, s) d s \leq-\alpha_{0}+\frac{\alpha_{0}}{8}+\frac{\alpha_{0}}{2^{4}}<-\frac{3 \alpha_{0}}{4},
$$

which proves the desired result. 
We deduce from (4.32) that $\forall t \in\left[-t_{1}, 0\right]$,

$$
\begin{aligned}
\frac{d}{d t}\left(\tilde{q}_{2}(t)-\tilde{q}_{1}(t)\right) & =\tilde{u}\left(\tilde{q}_{2}(t)\right)-\tilde{u}\left(\tilde{q}_{1}(t)\right) \\
& =\int_{\tilde{q}_{1}(t)}^{\tilde{q}_{2}(t)} \tilde{u}_{x}(t, s) d s \\
& \leq-\frac{\alpha_{0}}{2}\left(\tilde{q}_{2}(t)-\tilde{q}_{1}(t)\right) .
\end{aligned}
$$

Therefore,

$$
\left(\tilde{q}_{2}-\tilde{q}_{1}\right)(t) \geq\left(\tilde{q}_{2}-\tilde{q}_{1}\right)(0) e^{-\frac{\alpha_{0}}{2} t}=\beta e^{-\frac{\alpha_{0}}{2} t} .
$$

On the other hand, since according to (4.31) and (4.32), $\tilde{u}\left(t, \tilde{q}_{2}(t)\right) \geq 2 \alpha_{0} / 3$ and $\tilde{u}_{x} \leq 0$ on $] \tilde{q}_{1}(t), \tilde{q}_{2}(t)[$, we deduce that

$$
\tilde{u}\left(t, \tilde{q}_{1}(t)\right) \geq \tilde{u}\left(t, \tilde{q}_{2}(t)\right) \geq 2 \alpha_{0} / 3, \quad \text { on }\left[-t_{1}, 0\right] .
$$

Coming back to the solution $u$ emanating from $u_{0}$, it follows from (4.29) that

$$
\min \left(u\left(t, \tilde{q}_{1}\left(t_{1}\right)\right), u\left(t, \tilde{q}_{2}\left(t_{1}\right)\right)\right) \geq \frac{\alpha_{0}}{2} \text { with }\left(\tilde{q}_{2}-\tilde{q}_{1}\right)\left(t_{1}\right) \geq \beta e^{-\frac{\alpha_{0}}{2} t}, \quad \forall t \in\left[-t_{1}, 0\right] .
$$

Taking $t_{1}>0$ large enough, this contradicts the $Y$-almost localization of $u$ which proves that $a(t) \geq \frac{\alpha_{0}}{8}$ and thus $u_{x}(t, \cdot)$ has got a jump at $x(t)+x_{+}(t)$.

It is worth noticing that,according Lemma 4.1, this ensures that for all $t \in \mathbb{R}$, one can decompose $y(t)$ as

$$
y(t)=\nu(t)+a(t) \delta_{x(t)+x_{+}(t)}+\sum_{i=1}^{\infty} a_{i}(t) \delta_{x_{i}(t)}
$$

where $\nu(t)$ is a non negative non atomic measure with $\nu(t) \equiv 0$ on $] x(t)+x_{+}(t),+\infty[$, $\left\{a_{i}\right\}_{n \geq 1} \subset\left(\mathbb{R}_{+}\right)^{\mathbb{N}}$ with $\sum_{i=1}^{\infty} a_{i}(t)<\infty$ and $x_{i}(t)<x(t)+x_{+}(t)$ for all $i \in \mathbb{N}^{*}$.

It remains to prove that for all pair of real numbers $\left(t_{1}, t_{2}\right)$ with $t_{1}<t_{2}$,

$$
a\left(t_{2}\right)-a\left(t_{1}\right)=\frac{1}{2} \int_{t_{1}}^{t_{2}}\left(u^{2}-u_{x}^{2}\right)\left(\tau, q^{*}(\tau)-\right) d \tau .
$$

Indeed, since $\left|u_{x}\right| \leq u$ and $u \in C\left(\mathbb{R}^{2}\right)$ this will force $a$ to be non decreasing continuous function on $\mathbb{R}$. Then noticing that

$$
\begin{aligned}
\left(u^{2}-u_{x}^{2}\right)\left(t, q^{*}(t)-\right) & =a(t)\left(u-u_{x}\right)\left(t, q^{*}(t)-\right) \\
& =a(t)\left(2 u\left(t, q^{*}(t)-\right)-a(t)\right)=a(t)\left(2 u\left(t, q^{*}(t)\right)-a(t)\right)
\end{aligned}
$$

with $t \mapsto u\left(t, q^{*}(t)\right) \in C(\mathbb{R})$, the fundamental theorem of calculus will ensure that $a$ is differentiable on $\mathbb{R}$.

Let $\phi: \mathbb{R} \rightarrow \mathbb{R}_{+}$be a non decreasing $C^{\infty}$-function such that $\operatorname{supp} \phi \subset[-1,+\infty[$ and $\phi \equiv 1$ on $\mathbb{R}_{+}$. We set $\phi_{\varepsilon}=\phi(\dot{\bar{\varepsilon}})$. Since $u$ is continuous and $y(t, \cdot)=0$ on ]$x(t)+x_{+}(t),+\infty[$ it follows from (4.33) that for all $t \in \mathbb{R}$,

$$
a(t)=\lim _{\varepsilon \searrow 0}\left\langle y(t), \phi_{\varepsilon}\left(\cdot-q^{*}(t)\right)\right\rangle .
$$

Without loss of generality, it suffices to prove (4.34) for $t_{1}=0$ and $\left.t_{2}=t \in\right] 0,1[$. Let $\beta>0$ be fixed, we claim that there exists $\varepsilon_{0}>0$ such that for all $0<\varepsilon<\varepsilon_{0}$,

$\left.\left|\left\langle y(t), \phi_{\varepsilon}\left(\cdot-q^{*}(t)\right)\right\rangle-\left\langle y(0), \phi_{\varepsilon}\left(\cdot-q^{*}(0)\right)\right\rangle-\frac{1}{2} \int_{0}^{t} \int_{\mathbb{R}}\left(u^{2}-u_{x}^{2}\right)\left(\tau, q^{*}(t)+\varepsilon z\right) \phi^{\prime}(z) d z d \tau\right| \leq \beta, \quad \forall t \in\right] 0,1[$ 
Passing to the limit as $\varepsilon$ tends to 0 , this leads to the desired result. Indeed, since $\left(u^{2}-u_{x}^{2}\right)(\tau, \cdot) \in B V(\mathbb{R})$ and $\phi^{\prime} \equiv 0$ on $\mathbb{R}_{+}$, for any fixed $(\tau, z)$, it is clear that

$$
\left(u^{2}-u_{x}^{2}\right)\left(\tau, q^{*}(\tau)+\varepsilon z\right) \phi^{\prime}(z) \underset{\varepsilon \rightarrow 0}{\longrightarrow}\left(u^{2}-u_{x}^{2}\right)\left(\tau, q^{*}(\tau)-\right) \phi^{\prime}(z)
$$

and, since it is dominated by $2\left\|u_{0}\right\|_{H^{1}}^{2} \phi^{\prime}$, the dominated convergence theorem leads to

$$
\begin{aligned}
\left.\int_{0}^{t} \int_{\mathbb{R}}\left(u^{2}-u_{x}^{2}\right)\left(\tau, q^{*}(t)+\varepsilon z\right) \phi^{\prime}(z)\right) d z d \tau \quad \underset{\varepsilon \rightarrow 0}{\longrightarrow} & \left.\int_{0}^{t} \int_{\mathbb{R}}\left(u^{2}-u_{x}^{2}\right)\left(\tau, q^{*}(\tau)-\right) \phi^{\prime}(z)\right) d z d \tau \\
& =\int_{0}^{t}\left(u^{2}-u_{x}^{2}\right)\left(\tau, q^{*}(\tau)-\right) d \tau .
\end{aligned}
$$

To prove (4.35) we first notice that according to (4.33) for any $\alpha>0$ there exists $\gamma(\alpha)>0$ such that

$$
\|y(0)\|_{\mathcal{M}(] q^{*}(0)-\gamma(\alpha), q^{*}(0)[)}<\alpha .
$$

We take $\varepsilon_{0}=\gamma\left(\frac{\beta}{2} e^{-2\left\|u_{0}\right\|_{H^{1}}}\right)$. As above, we approximate again $u(0)$ by a sequence $\left\{u_{0, n}\right\} \subset H^{\infty}(\mathbb{R}) \cap Y_{+}$such that $\left\|u_{0, n}\right\|_{H^{1}} \leq 2\left\|u_{0}\right\|_{H^{1}}$ and

$$
\left\|y(0)-y_{0, n}\right\|_{\mathcal{M}(\mathbb{R})} \leq \beta / 4 \text {. }
$$

where $y_{0, n}=u_{0, n}-\partial_{x}^{2} u_{0, n}$. We again denote respectively by $u_{n}$ and $y_{n}$, the solution to (1.5) emanating from $u_{0, n}$ and its momentum density $u_{n}-u_{n, x x}$. Let now $q_{n}^{*}: \mathbb{R} \rightarrow \mathbb{R}$ be the integral line of $u_{n}$ defined by $q_{n}^{*}(t)=q_{n}\left(t, q^{*}(0)\right)$ where $q_{n}$ is defined in (4.10). On account of (1.4), it holds

$$
\begin{aligned}
\frac{d}{d t} \int_{\mathbb{R}} y_{n} \phi_{\varepsilon}\left(\cdot-q_{n}^{*}(t)\right)= & -u_{n}\left(t, q_{n}^{*}(t)\right) \int_{\mathbb{R}} y_{n} \phi_{\varepsilon}^{\prime}-\int_{\mathbb{R}} \partial_{x}\left(y_{n} u_{n}\right) \phi_{\varepsilon}-\int_{\mathbb{R}} y_{n} u_{n, x} \phi_{\varepsilon} \\
= & \int_{\mathbb{R}}\left[u_{n}(t, \cdot)-u_{n}\left(t, q^{*}(t)\right)\right] y_{n}(t, \cdot) \phi_{\varepsilon}^{\prime}+\frac{1}{2} \int_{\mathbb{R}}\left(u_{n}^{2}(t, \cdot)-u_{n, x}^{2}(t, \cdot)\right) \phi_{\varepsilon}^{\prime} \\
= & \frac{1}{\varepsilon} \int_{\mathbb{R}}\left[u_{n}(t, \cdot)-u_{n}\left(t, q^{*}(t)\right)\right] y_{n}(t, \cdot) \phi^{\prime}\left(\frac{\cdot-q_{n}^{*}(t)}{\varepsilon}\right) \\
& +\frac{1}{2} \int_{\mathbb{R}}\left(u_{n}^{2}-u_{n, x}^{2}\right)\left(t, q_{n}^{*}(t)+\varepsilon z\right) \phi^{\prime}(z) d z \\
= & I_{t}^{\varepsilon, n}+I I_{t}^{\varepsilon, n} .
\end{aligned}
$$

Since, according to $(2.2),\left|u_{n, x}\right| \leq\left\|u_{0, n}\right\|_{H^{1}} \leq 2\left\|u_{0}\right\|_{H^{1}}$,

$$
\begin{aligned}
\left|I_{t}^{\varepsilon, n}\right| & \leq \frac{2\left\|u_{0}\right\|_{H^{1}}}{\varepsilon} \int_{\mathbb{R}}\left|x-q_{n}^{*}(t)\right| y_{n}(t, x) \phi^{\prime}\left(\frac{x-q_{n}^{*}(t)}{\varepsilon}\right) d x \\
& \leq 2\left\|u_{0}\right\|_{H^{1}} \int_{\mathbb{R}} y_{n}(t, x) \phi^{\prime}\left(\frac{x-q_{n}^{*}(t)}{\varepsilon}\right) d x
\end{aligned}
$$

Now, in view of (4.16) we easily get

$$
\left.e^{-2\left\|u_{0}\right\|_{H^{1}}} \leq q_{n, x}(t, z) \leq e^{2\left\|u_{0}\right\|_{H^{1}}}, \quad \forall(t, z) \in\right]-1,1[\times \mathbb{R}
$$


and the change of variables $x=q_{n}(t, z)$ together with the identity (4.11) lead to

$$
\begin{aligned}
\int_{\mathbb{R}} y_{n}(t, x) \phi^{\prime}\left(\frac{x-q_{n}^{*}(t)}{\varepsilon}\right) d x & =\int_{\mathbb{R}} y_{n}\left(t, q_{n}(t, z)\right) q_{n}^{\prime}(t, z) \phi^{\prime}\left(\frac{q_{n}(t, z)-q_{n}^{*}(t)}{\varepsilon}\right) d x \\
& \leq e^{2\left\|u_{0}\right\|_{H^{1}}} \int_{\mathbb{R}} y_{n}\left(t, q_{n}(t, z)\right)\left(q_{n}^{\prime}(t, z)\right)^{2} \phi^{\prime}\left(\frac{q_{n}(t, z)-q_{n}^{*}(t)}{\varepsilon}\right) d z \\
& \leq e^{2\left\|u_{0}\right\|_{H^{1}}} \int_{\mathbb{R}} y_{n}(0, z) \phi^{\prime}\left(\frac{q_{n}(t, z)-q_{n}^{*}(t)}{\varepsilon}\right) d z .
\end{aligned}
$$

But, making use of the mean value theorem, (4.39) and the definition of $\phi$, we obtain that, for any $t \in[0,1], z \mapsto \phi^{\prime}\left(\frac{q_{n}(t, z)-q_{n}^{*}(t)}{\varepsilon}\right)$ is supported in an interval of length at most $\varepsilon e^{2\left\|u_{0}\right\|_{H^{1}}}$. Therefore, according to (4.36) and (4.37), setting $\varepsilon_{0}=e^{-2\left\|u_{0}\right\|_{H^{1}}} \gamma\left(\frac{\beta}{2} e^{-2\left\|u_{0}\right\|_{H^{1}}}\right)$, it follows that for all $0<\varepsilon<\varepsilon_{0}$ and all $n \in \mathbb{N}$,

$$
\int_{0}^{t}\left|I_{\tau}^{\varepsilon, n}\right| d \tau \leq 3 \beta / 4
$$

To estimate the contribution of $I I_{t}^{\varepsilon, n}$ we first notice that thanks (2.4) it holds

$$
u_{n, x} \rightarrow u_{x} \text { in } C\left([-1,1] ; L^{2}(\mathbb{R})\right)
$$

and for all $t \in[-1,1]$, Helly's theorem ensures that

$$
u_{n, x}(t, \cdot) \rightarrow u_{x}(t, \cdot) \text { a.e. on } \mathbb{R} .
$$

Hence, for any fixed $t \in[-1,1]$ there exists a set $\Omega_{t} \subset \mathbb{R}$ of Lebesgue measure zero such that $u_{x}(t)$ is continuous at every point $x \in \mathbb{R} / \Omega_{t}$ and

$$
u_{n, x}(t, x) \rightarrow u_{x}(t, x), \quad \forall x \in \mathbb{R} / \Omega_{t} .
$$

Since $q_{n}^{*}(t) \rightarrow q^{*}(t)$, it follows that

$$
u_{n, x}\left(t, q_{n}^{*}(t)+x\right) \rightarrow u_{x}\left(t, q^{*}(t)+x\right), \quad \forall x \in \mathbb{R} / \tau_{q^{*}(t)}\left(\Omega_{t}\right) .
$$

where for any set $\Lambda \subset \mathbb{R}$ and any $a \in \mathbb{R}, \tau_{a}(\Lambda)=\{x-a, a \in \Lambda\}$.

Since the integrand in $I I_{t}^{\varepsilon, n}$ is bounded by $2\left\|u_{0}\right\|_{H^{1}} \phi^{\prime} \in L^{1}(\mathbb{R})$, it follows from Lebesgue dominated convergence theorem that for any $t \in[-1,1]$,

$$
I I_{t}^{\varepsilon, n} \underset{n \rightarrow \infty}{\longrightarrow} \frac{1}{2} \int_{\mathbb{R}}\left(u^{2}-u_{x}^{2}\right)\left(t, q^{*}(t)+\varepsilon z\right) \phi^{\prime}(z) d z .
$$

Therefore, invoking again Lebesgue dominated convergence theorem, but on $] 0, t[$, keeping in mind that $\left\{\left|u_{n}\right|\right\} \mid$ and $\left\{\left|u_{n, x}\right|\right\}$ are uniformly bounded on $\mathbb{R}^{2}$ by $2\left\|u_{0}\right\|_{H^{1}}$, we finally deduce that for any fixed $t \in] 0,1[$,

$$
\left.\int_{0}^{t} I I_{\tau}^{\varepsilon, n} d \tau \underset{n \rightarrow \infty}{\longrightarrow} \frac{1}{2} \int_{0}^{t} \int_{\mathbb{R}}\left(u^{2}-u_{x}^{2}\right)\left(\tau, q^{*}(t)+\varepsilon z\right) \phi^{\prime}(z)\right) d z d \tau
$$

Now, we fix $t \in] 0,1[$ and $\varepsilon \in] 0, \varepsilon_{0}[$. According to the convergence result (2.5), for $n$ large enough it holds

$$
\left|\left\langle y_{n}(t)-y(t), \phi_{\varepsilon}\left(\cdot-q_{*}(t)\right)\right\rangle\right|+\mid\left\langle y_{n}\left(0-y(0), \phi_{\varepsilon}\left(\cdot-q_{*}(0)\right)\right\rangle\right| \leq \beta / 4 .
$$

which together with (4.38) and (4.40)-(4.41) prove the claim (4.35).

Lemma 4.5. There exists $\left(a_{-}, a_{+}\right) \in\left[\frac{\alpha_{0}}{8}, 2\left\|u_{0}\right\|_{H^{1}}\right]^{2}$, with $a_{-} \leq a_{+}$such that

$$
\begin{aligned}
& \lim _{t \rightarrow+\infty} u\left(t, x(t)+x_{+}(t)\right)=\lim _{t \rightarrow+\infty} a(t) / 2=a_{+} / 2, \\
& \lim _{t \rightarrow-\infty} u\left(t, x(t)+x_{+}(t)\right)=\lim _{t \rightarrow-\infty} a(t) / 2=a_{-} / 2,
\end{aligned}
$$


Proof. The existence of the limits at $\mp \infty$ for $a(\cdot)$ follows from the monotonicity of $a(\cdot)$. Now, in view of Proposition 4.4, for all $t \in \mathbb{R}$,

$$
\begin{aligned}
0 \leq a^{\prime}(t)=\frac{1}{2}\left(u^{2}-u_{x}^{2}\right)\left(t, x(t)+x_{+}(t)-\right) & =\frac{a(t)}{2}\left(u-u_{x}\right)\left(t, x(t)+x_{+}(t)-\right) \\
& =\frac{a(t)}{2}\left(2 u\left(t, x(t)+x_{+}(t)\right)-a(t)\right) .
\end{aligned}
$$

Therefore, since $a$ takes values in $\left[\alpha_{0} / 8,2\left\|u_{0}\right\|_{H^{1}}\right]$, it remains to prove that $a^{\prime}(t) \rightarrow 0$ as $t \rightarrow \pm \infty$. Since

$$
\int_{\mathbb{R}} a^{\prime}(\tau) d \tau<\infty
$$

the desired result will follow if $a^{\prime}$ is Lipschitz on $\mathbb{R}$. But this is not too hard to check. Indeed, first from (4.25) we have for all $t \in \mathbb{R},|a(t)-a(0)| \leq t\left\|u_{0}\right\|_{H^{1}}^{2}$ and thus $t \mapsto a(t)$ is clearly Lipschitz on $\mathbb{R}$. Second, since $x(t)+x_{+}(t)=q^{*}(t)$, it holds

$$
\frac{d}{d t} u\left(t, x(t)+x_{+}(t)\right)=u\left(t, q^{*}(t)\right) u_{x}\left(t, q^{*}(t)\right)+u_{t}\left(t, q^{*}(t)\right) .
$$

But, $\sup _{(t, x) \in \mathbb{R}^{2}}\left|u u_{x}\right| \leq 2\left\|u_{0}\right\|_{H^{1}}^{2}$ and

$$
\begin{aligned}
\sup _{(t, x) \in \mathbb{R}^{2}}\left|u_{t}\right| & \leq \sup _{(t, x) \in \mathbb{R}^{2}}\left|u u_{x}\right|+\sup _{(t, x) \in \mathbb{R}^{2}}\left|\left(1-\partial_{x}^{2}\right)^{-1} \partial_{x}\left(u^{2}+\frac{1}{2} u_{x}^{2}\right)\right| \\
& \lesssim\left\|u_{0}\right\|_{H^{1}}^{2}+\sup _{t \in \mathbb{R}}\left\|u^{2}+u_{x}^{2}\right\|_{L_{x}^{2}} \\
& \lesssim\left\|u_{0}\right\|_{H^{1}}^{2} .
\end{aligned}
$$

Therefore $t \mapsto u\left(t, x(t)+x_{+}(t)\right)$ is also Lipschitz on $\mathbb{R}$ which achieves the proof thanks to (4.44).

4.3. End of the proof of Theorem 1.1. In this subsection, we conclude by proving that the jump of $u_{x}(0, \cdot)$ at $x(0)+x_{+}(0)$ is equal to $-2 u\left(0, x(0)+x_{+}(0)\right)$. This saturates for all $v \in Y_{+}$, the relation between the jump of $v_{x}$ and the value of $v$ at a point $\xi \in \mathbb{R}$ and forces $u(0, \cdot)$ to be equal to $u\left(0, x(0)+x_{+}(0)\right) \varphi\left(\cdot-x(0)+x_{+}(0)\right)$.

We use the invariance of the $(\mathrm{CH})$ equation under the transformation $(t, x) \mapsto$ $(-t,-x)$. This invariance ensures that $v(t, x)=u(-t,-x)$ is also a solution of the $(\mathrm{C}-\mathrm{H})$ equation that belongs to $C\left(\mathbb{R} ; H^{1}(\mathbb{R})\right.$, with $u-u_{x x} \in C_{w}\left(\mathbb{R} ; \mathcal{M}_{+}\right)$and shares the property of $Y$-almost localization with $x(\cdot)$ replaced by $-x(-\cdot)$ and the same fonction $\varepsilon \mapsto R_{\varepsilon}$ (See Definition 1.1). Therefore, by applying Propositions $4.2,4.4$ and Lemma 4.3 for $v$ we infer that there exists a $C^{1}$-function $x_{-}: \mathbb{R} \mapsto$ ]$\left.-\infty, r_{0}\right]$ and a derivable non decreasing function $\tilde{a}: \mathbb{R} \rightarrow\left[\alpha_{0} / 8,2\left\|u_{0}\right\|_{H^{1}}\right]$ with $\lim _{t \rightarrow \mp \infty} \tilde{a}(t)=\tilde{a}_{\mp}$ such that

$$
\tilde{a}(t)=v_{x}\left(t,\left(-x(-t)+x_{+}(t)\right)+\right)-v_{x}\left(t,\left(-x(-t)+x_{+}(t)\right)-\right), \quad \forall t \in \mathbb{R} .
$$

Moreover,

$$
\lim _{t \rightarrow \mp \infty} v\left(t,-x(-t)+x_{+}(t)\right)=\lim _{t \rightarrow \mp \infty} \tilde{a}(t) / 2=\tilde{a}_{\mp} / 2 .
$$

Coming back to $u$ this ensures that

$$
\begin{aligned}
\lim _{t \rightarrow+\infty} u\left(t, x(t)-x_{-}(-t)\right) & =\lim _{t \rightarrow-\infty} \tilde{a}(t) / 2=\tilde{a}_{-} / 2, \\
\lim _{t \rightarrow-\infty} u\left(t, x(t)-x_{-}(-t)\right) & =\lim _{t \rightarrow+\infty} \tilde{a}(t) / 2=\tilde{a}_{+} / 2,
\end{aligned}
$$


At this stage let us underline that since

$$
x_{-}(-t)=\sup \{x \in \mathbb{R}, \operatorname{supp} y(-t) \in[x(t)-x(-t),+\infty[\}
$$

and $u \not \equiv 0$ we must have $x(-t)+x(t) \geq 0$ for all $t \in \mathbb{R}$. We claim that this forces

$$
\tilde{a}_{-}=\tilde{a}_{+}=a_{-}=a_{+} .
$$

Note first that since $\tilde{a}_{-} \leq \tilde{a}_{+}$and $a_{-} \leq a_{+}$, it suffices to prove that $\tilde{a}_{-} \geq a_{+}$ and $\tilde{a}_{+} \leq a_{-}$. This follows easily by a contradiction argument. Indeed, assume for instance that $\tilde{a}_{-}<a_{+}$. Then, there exists $t_{0} \in \mathbb{R}$ and $\varepsilon>0$ such that $u(t, x(t)-$ $\left.x_{-}(-t)\right)<u\left(t, x(t)+x_{+}(t)\right)-\varepsilon$ for all $t \geq t_{0}$. Since $x(t)-x_{-}(-t)=q\left(t-t_{0}, x\left(t_{0}\right)-\right.$ $\left.x_{-}\left(-t_{0}\right)\right)$ and $x(t)+x_{+}(t)=q\left(t-t_{0}, x\left(t_{0}\right)+x_{+}\left(t_{0}\right)\right)$, it follows from (4.22) that

$$
\left.x_{+}(t)+x_{-}(-t)\right) \geq \varepsilon\left(t-t_{0}\right) \underset{t \rightarrow+\infty}{\longrightarrow}+\infty
$$

which contradicts that $\left.\left.\left(x_{+}(t), x_{-}(t)\right) \in\right]-\infty, r_{0}\right]^{2}$. Exactly the same argument but with $t \rightarrow-\infty$ ensures that $\tilde{a}_{+} \leq a_{-}$and completes the proof of the claim (4.48).

We deduce from (4.48) that $a(t)=a+$ for all $t \in \mathbb{R}$ and thus (4.44), (4.21) and (4.24) force

$$
u\left(t, x(0)+x_{+}(0)+\frac{a_{+}}{2} t\right)=\frac{a_{+}}{2}, \quad \forall t \in \mathbb{R}
$$

and

$$
u_{x}\left(t,\left(x(0)+x_{+}(0)+\frac{a_{+}}{2} t\right)-\right)-u_{x}\left(t,\left(x(0)+x_{+}(0)+\frac{a_{+}}{2} t\right)+\right)=a_{+}, \quad \forall t \in \mathbb{R} .
$$

In particular, in view of (4.33),

$$
u\left(0, x(0)+x_{+}(0)\right)=\frac{a_{+}}{2} \text { and } y(0)=a_{+} \delta_{x(0)+x_{+}(0)}+\mu
$$

for some $\mu \in \mathcal{M}_{+}(\mathbb{R})$. But this forces $\mu=0$ since

$$
\left(1-\partial_{x}^{2}\right)^{-1}\left(a_{+} \delta_{x(0)+x_{+}(0)}\right)=\frac{a_{+}}{2} \exp \left(-\left|\cdot-\left(x(0)+x_{+}(0)\right)\right|\right)
$$

and for any $\mu \in \mathcal{M}_{+}(\mathbb{R})$, with $\mu \neq 0$, it holds

$$
\left(1-\partial_{x}^{2}\right)^{-1} \nu=\frac{1}{2} e^{-|x|} * \nu>0 \text { on } \mathbb{R} .
$$

We thus conclude that $y(0)=a_{+} \delta_{x(0)+x_{+}(0)}$ which leads to

$$
u(t, x)=\frac{a_{+}}{2} \exp \left(-\left|x-x(0)-x_{+}(0)-\frac{a_{+}}{2} t\right|\right)
$$

\section{Asymptotic stability of the PeAkon}

Let $c>0$ and $u_{0} \in Y_{+}$such that

$$
\left\|u_{0}-c \varphi\right\|_{H^{1}}<\left(\frac{\varepsilon^{2}}{3 c^{2}}\right)^{4}, \quad 0<\varepsilon<c,
$$

then, according to [18],

$$
\sup _{t \in \mathbb{R}}\|u(t)-c \varphi(\cdot-\xi(t))\|_{H^{1}}<\frac{\varepsilon^{2}}{c},
$$

where $u \in C\left(\mathbb{R} ; H^{1}\right)$ is the solution emanating from $u_{0}$ and $\xi(t) \in \mathbb{R}$ is any point where the function $u(t, \cdot)$ attains its maximum. By the implicit function theorem, 
one can prove the following lemma (see for instance ${ }^{5}[21]$ ) whose proof is postponed to the appendix.

Lemma 5.1. There exists $0<\varepsilon_{0}<1, \kappa_{0}>0, n_{0} \in \mathbb{N}$ and $K>1$ such that if a solution $u \in C(\mathbb{R} ; Y)$ to $(1.5)$ satisfies

$$
\sup _{t \in \mathbb{R}}\|u(t)-c \varphi(\cdot-z(t))\|_{H^{1}}<c \varepsilon_{0}
$$

for some function $z: \mathbb{R} \rightarrow \mathbb{R}$, then there exists a unique function $x: \mathbb{R} \rightarrow \mathbb{R}$ such that

$$
\sup _{t \in \mathbb{R}}|x(t)-z(t)|<\kappa_{0}
$$

and

$$
\int_{\mathbb{R}} u(t)\left(\rho_{n_{0}} * \varphi^{\prime}\right)(\cdot-x(t))=0, \quad \forall t \in \mathbb{R},
$$

where $\left\{\rho_{n}\right\}$ is defined in (2.3) and where $n_{0}$ satisfies :

$$
\forall y \in[-1 / 2,1 / 2], \quad \int_{\mathbb{R}} \varphi(\cdot-y)\left(\rho_{n_{0}} * \varphi^{\prime}\right)=0 \Leftrightarrow y=0 .
$$

Moreover, $x(\cdot) \in C^{1}(\mathbb{R})$ with

$$
\sup _{t \in \mathbb{R}}|\dot{x}(t)-c| \leq \frac{c}{8}
$$

and if

$$
\sup _{t \in \mathbb{R}}\|u(t)-c \varphi(\cdot-z(t))\|_{H^{1}}<\frac{\varepsilon^{2}}{c}=c\left(\frac{\varepsilon}{c}\right)^{2}
$$

for $0<\varepsilon<c \varepsilon_{0}$ then

$$
\sup _{t \in \mathbb{R}}\|u(t)-c \varphi(\cdot-x(t))\|_{H^{1}} \leq K \varepsilon .
$$

At this stage, we fix $0<\theta<c$ and we take

$$
\varepsilon=\frac{1}{2 K} \min \left(\frac{\theta}{2^{8}}, c \varepsilon_{0}\right)
$$

For $u_{0} \in Y_{+}$satisfying (5.1) with this $\varepsilon$, (5.2) ensures that (5.3) and thus (5.7) hold. Moreover, (5.9) is satisfies with

$$
K \varepsilon \leq \min \left(\frac{\theta}{2^{9}}, \frac{c \varepsilon_{0}}{2}\right)
$$

It follows that $\dot{x} \geq \frac{3}{4} c$ on $\mathbb{R}$ and that $u$ satisfies the hypotheses of Lemma 3.2 for any $0<\alpha<1$ such that

$$
(1-\alpha) \geq \frac{\theta}{4 c}
$$

and any $0 \leq \gamma \leq(1-\alpha) c$. In particular, $u$ satisfies the hypotheses of Lemma 3.2 for $\alpha=1 / 3$. Note that the hypothesis (1.8) with

$$
\eta_{0}=\frac{1}{K^{8}} \min \left(\frac{1}{2^{10}}, \frac{\varepsilon_{0}}{6}\right)^{8}
$$

\footnotetext{
${ }^{5}$ In [21], this lemma is stated with $\varphi^{\prime}$ instead of $\rho_{n_{0}} * \varphi^{\prime}$ in (5.5). However, there is a gap in the proof since the non smoothness of $\varphi$ makes the $C^{1}$ regularity of $x(\cdot)$ difficult to prove with this orthogonality condition.
} 
implies that (5.1) holds with $\varepsilon$ given by (5.10).

In the sequel we will make use of the following functionals that measure the quantity $E(u)+\gamma M(u)$ at the right and at the left of $u$. For $0 \leq \gamma \leq \frac{2 c}{3}, v \in Y$ and $R>0$ we set

$$
J_{\gamma, r}^{R}(v)=\left\langle v^{2}+v_{x}^{2}+\gamma\left(v-v_{x x}\right), \Psi(\cdot-R)\right\rangle .
$$

and

$$
J_{\gamma, l}^{R}(v)=\left\langle v^{2}+v_{x}^{2}+\gamma\left(v-v_{x x}\right),(1-\Psi(\cdot+R))\right\rangle
$$

Let $t_{0} \in \mathbb{R}$ be fixed. Fixing $\alpha=\beta=1 / 3$ and taking $z(\cdot)=(1-\alpha) x(\cdot), z(\cdot)$ clearly satisfies (3.6). Moreover, we have $J_{\gamma, r}^{R}\left(u\left(t_{0}, \cdot+x\left(t_{0}\right)\right)=I_{t_{0}}^{+R}\left(t_{0}\right)\right.$ where $I_{t_{0}}^{+R}$ is defined in (3.7). Since obviously,

$$
J_{\gamma, r}^{R}(u(t, \cdot+x(t))) \geq I_{t_{0}}^{+R}(t), \quad \forall t \leq t_{0},
$$

we deduce from (3.8) that

$$
J_{\gamma, r}^{R}\left(u\left(t_{0}, \cdot+x\left(t_{0}\right)\right)\right) \leq J_{\gamma, r}^{R}(u(t, \cdot+x(t)))+K_{0} e^{-R / 6}, \quad \forall t \leq t_{0},
$$

where $K_{0}$ is the constant appearing in (3.8). Now, let us define

$$
\begin{aligned}
\tilde{I}_{t_{0}}^{R}(t) & =\left\langle u^{2}(t)+u_{x}^{2}(t)+c y(t), 1-\Psi\left(\cdot-x(t)+R+\alpha\left(x\left(t_{0}\right)-x(t)\right)\right)\right\rangle \\
& =E(u(t))+c M(u(t))-I_{t_{0}}^{-R}(t),
\end{aligned}
$$

where we take again $z(\cdot)=(1-\alpha) x(\cdot)$. Since $M(\cdot)$ and $E(\cdot)$ are conservation laws, (3.9) leads to

$$
\tilde{I}_{t_{0}}^{R}(t) \geq \tilde{I}_{t_{0}}^{R}\left(t_{0}\right)-C e^{-R / 6}, \forall t \geq t_{0} .
$$

We thus deduce as above that $\forall t \geq t_{0}$,

$$
J_{\gamma, l}^{R}(u(t, \cdot+x(t))) \geq J_{\gamma, l}^{R}\left(x\left(t_{0}, \cdot+x\left(t_{0}\right)\right)\right)-K_{0} e^{-R / 6} .
$$

The following proposition proved in the appendix ensures that, for $\varepsilon$ small enough, the $\omega$-limit set for the weak $H^{1}$-topology of the orbit of $u_{0}$ is constituted by initial data of $Y$-almost localized solutions. The crucial tools in the proof are the almost monotonicity properties (5.14) and (5.15).

Proposition 5.2. Let $u_{0} \in Y_{+}$satisfying (5.1) with $\varepsilon$ defined as in (5.10) and let $u \in C\left(\mathbb{R} ; H^{1}(\mathbb{R})\right)$ be the solution of $(1.5)$ emanating from $u_{0}$. For any sequence $t_{n} \nearrow+\infty$ there exists a subsequence $\left\{t_{n_{k}}\right\} \subset\left\{t_{n}\right\}$ and $\tilde{u}_{0} \in Y_{+}$such that

$$
u\left(t_{n_{k}}, \cdot+x\left(t_{n_{k}}\right)\right) \underset{n_{k} \rightarrow+\infty}{\rightarrow} \tilde{u}_{0} \text { in } H^{1}(\mathbb{R})
$$

and

$$
u\left(t_{n_{k}}, \cdot+x\left(t_{n_{k}}\right)\right) \underset{n_{k} \rightarrow+\infty}{\longrightarrow} \tilde{u}_{0} \text { in } H_{l o c}^{1}(\mathbb{R})
$$

where $x(\cdot)$ is a $C^{1}$-function satisfying (5.5), (5.7) and (5.9). Moreover, the solution of (1.5) emanating from $\tilde{u}_{0}$ is $Y$-almost localized.

So, let $u_{0} \in Y_{+}$satisfying (5.1) with $\varepsilon$ defined as in (5.10) and let $t_{n} \nearrow+\infty$ be a sequence of positive real numbers. According to the above proposition, (5.16)(5.17) hold for some subsequence $\left\{t_{n_{k}}\right\} \subset\left\{t_{n}\right\}$ and $\tilde{u}_{0} \in Y_{+}$such that the solution of (1.5) emanating from $\tilde{u}_{0}$ is $Y$-almost localized. Theorem 1.1 then forces

$$
\tilde{u}_{0}=c_{0} \varphi\left(\cdot-x_{0}\right)
$$


for some $x_{0} \in \mathbb{R}$ and $c_{0}$ such that $\left|c-c_{0}\right| \leq K \varepsilon \leq c / 2^{9}$. Note that (5.16) together with (5.9) imply $\left\|c_{0} \varphi\left(\cdot-x_{0}\right)-c \varphi\right\|_{H^{1}} \leq K \varepsilon$ and thus (5.10) and (5.17) ensure that $\left|x_{0}\right| \ll 1 / 2$. Since by (5.16), $\tilde{u}_{0}$ satisfies the orthogonality condition (5.5), (5.6) then forces $x_{0}=0$. On the other hand, (5.17) and (5.9) ensure that $c_{0}=$ $\lim _{n \rightarrow+\infty} \max _{\mathbb{R}} u\left(t_{n_{k}}\right)$ and thus

$$
u\left(t_{n_{k}}, \cdot+x\left(t_{n_{k}}\right)\right)-\lambda\left(t_{n_{k}}\right) \varphi \underset{k \rightarrow+\infty}{\rightarrow} 0 \text { in } H^{1}(\mathbb{R})
$$

where we set $\lambda(t):=\max _{\mathbb{R}} u(t), \quad \forall t \in \mathbb{R}$. Since this is the only possible limit, it follows that

$$
u(t, \cdot+x(t))-\lambda(t) \varphi \underset{t \rightarrow+\infty}{\rightarrow} 0 \text { in } H^{1}(\mathbb{R})
$$

and thus

$$
u(t, \cdot+x(t))-\lambda(t) \varphi \underset{t \rightarrow 0}{\longrightarrow} 0 \text { in } H_{l o c}^{1}(\mathbb{R})
$$

5.1. Convergence in $H^{1}(]-A,+\infty[)$ for any $A>0$. Let $\delta>0$ be fixed. Choosing $R>0$ such that $J_{0, r}^{R}(u(0), \cdot+x(0))<\delta$ and $K_{0} e^{-R / 6} \leq \delta$, where $K_{0}$ is the constant that appears in (5.14). We deduce from (5.14) that $J_{0, r}^{R}(u(t, \cdot+x(t)))<2 \delta$ for all $t \geq 0$. This fact together with the local strong convergence (5.17) clearly ensure that

$$
u(t, \cdot+x(t))-\lambda(t) \varphi \underset{t \rightarrow+\infty}{\longrightarrow} 0 \text { in } H^{1}(]-A,+\infty[) \text { for any } A>0
$$

5.2. Convergence of the scaling parameter. We claim that

$$
\lambda(t) \underset{t \rightarrow+\infty}{\longrightarrow} c_{0} .
$$

Let us fix again $\delta>0$ and take $R>0$ such that $K_{0} e^{-R / 6}<\delta$. (5.15) with $\gamma=0$ together with the conservation of $E(u)$ ensure that, for any pair $\left(t, t^{\prime}\right) \in \mathbb{R}^{2}$ with $t>t^{\prime}$ it holds

$$
\int_{\mathbb{R}}\left(u^{2}+u_{x}^{2}\right)(t, x) \Psi(x-x(t)+R) d x \leq \int_{\mathbb{R}}\left(u^{2}+u_{x}^{2}\right)\left(t^{\prime}, x\right) \Psi\left(x-x\left(t^{\prime}\right)+R\right) d x+\delta
$$

On the other hand, by the strong convergence (5.19) and the exponential localization of $\varphi, \varphi^{\prime}$ and $\Psi$, there exists $T>0$ such that for all $t \geq T$,

$$
\left|\int_{\mathbb{R}}\left(u^{2}+u_{x}^{2}\right)(t, x) \Psi(x-x(t)+R) d x-\lambda^{2}(t) E(\varphi)\right| \leq \delta .
$$

It thus follows that

$$
\lambda^{2}(t) E(\varphi) \leq \lambda^{2}\left(t^{\prime}\right) E(\varphi)+3 \delta, \quad \forall t>t^{\prime}>T .
$$

Since $\delta>0$ is arbitrary, this forces $\lambda$ to have a limit at $+\infty$ and completes the proof of the claim. 
5.3. Convergence of $\dot{x}$. We set $W(t, \cdot):=c_{0} \varphi(\cdot-x(t))$ and $\eta(t)=u(t)-c_{0} \varphi(\cdot-$ $x(t))=u(t)-W(t)$ for all $t \geq 0$. Differentiating (5.5) with respect to time and using that $\varphi-\varphi^{\prime \prime}=2 \delta_{0}$, we get

$$
\int_{\mathbb{R}} \eta_{t} \partial_{x} W=\dot{x}\left\langle\partial_{x}^{2} W, \eta\right\rangle_{H^{-1}, H^{1}}=-2 c_{0} \dot{x} \eta(x(t))+\dot{x} \int_{\mathbb{R}} \eta W
$$

and thus

$$
\left|\int_{\mathbb{R}} \eta_{t} \partial_{x} W\right| \leq 3 c_{0}\left|\dot{x}-c_{0}\right|\|\eta\|_{H^{1}}+2 c_{0}^{2}|\eta(x(t))|+c_{0}\left|\int_{\mathbb{R}} \eta W\right| .
$$

Substituting $u$ by $\eta+W$ in (1.5) and using the equation satisfied by $W$, we obtain the following equation satisfied by $v$ :

$$
\eta_{t}-\left(\dot{x}-c_{0}\right) \partial_{x} W=-\partial_{x} \eta W-\left(1-\partial_{x}^{2}\right)^{-1} \partial_{x}\left(2 \eta W+\eta_{x} W_{x}\right) .
$$

At this stage it is worth noticing that (5.19)-(5.20) ensures that

$$
|\eta(x(t))|+\left\|\eta_{x}(t) W(t)\right\|_{L^{2}}+\|\eta(t) W(t)\|_{L^{2}}+\left\|\eta_{x}(t) W_{x}(t)\right\|_{L^{2}} \underset{t \rightarrow+\infty}{\longrightarrow} 0 .
$$

Taking the $L^{2}$-scalar product with $\partial_{x} W$, integrating by parts, using that $\left\|\partial_{x} W\right\|_{L^{2}}^{2}=$ $c_{0}^{2}$ and the decay of $\varphi$ and its first derivative, (5.21), (5.22), (5.9) and the definition of $\varepsilon$, we get

$$
\left|\dot{x}(t)-c_{0}\right|\left(c_{0}^{2}-3 c_{0} \frac{c}{2^{8}}\right) \underset{t \rightarrow \infty}{\longrightarrow} 0 .
$$

This yields the desired result since $\left|c-c_{0}\right| \leq K \varepsilon=\frac{c}{2^{8}}$ clearly forces $c \leq 2 c_{0}$.

5.4. Strong $H^{1}$-convergence on $] \theta t,+\infty[$. We deduce from (5.20) that

$$
u(t, \cdot)-c_{0} \varphi(\cdot-x(t)) \underset{t \rightarrow+\infty}{\rightarrow} 0 \text { in } H^{1}(\mathbb{R})
$$

and

$$
u(t, \cdot+x(t))-c_{0} \varphi \underset{t \rightarrow+\infty}{\longrightarrow} 0 \text { in } H^{1}(]-A,+\infty[) \text { for any } A>0 .
$$

(1.10) will follow by combining these convergence results with the almost non increasing property (3.8). Indeed, let us fix $\delta>0$ and take $R \gg 1$ such that

$$
\|\varphi\|_{H^{1}(]-\infty,-R / 2[}^{2}<\delta \text { and }\|\Psi-1\|_{L^{\infty}(] R / 2,+\infty[)}<\delta
$$

where $\Psi$ is defined in (3.3). According to the above convergence result there exists $t_{0}>0$ such that $x\left(t_{0}\right)>R$ and for all $t \geq t_{0}$,

$$
\left\|\left(\eta^{2}+\eta_{x}^{2}\right)(t, \cdot+x(t))\right\|_{H^{1}(]-R / 2,+\infty[)}<\delta,
$$

where we set $\eta=u(t)-c_{0} \varphi(\cdot-x(t))$. In particular, (5.24) ensures that

$$
\left|E(\varphi)-\int_{\mathbb{R}}\left(u(t, \cdot+x(t)) \varphi+u_{x}(t, \cdot+x(t)) \varphi_{x}\right) \Psi(\cdot+y)\right| \lesssim \delta, \quad \forall y \geq R, \forall t \geq t_{0},
$$

We set $z(t)=\frac{\theta}{2} t$ and notice that (5.11) ensures that (3.6) is satisfied with $1-\alpha=\frac{\theta}{4 c}$ and $\beta=1 / 4$. Moreover, as noticing in the beginning of this section (see (5.11)), $u$ satisfies the hypotheses of Lemma (3.2) for such $\alpha$. According to (3.9) with $\gamma=0$, we thus get for all $t \geq t_{0}$,

$$
\int_{\mathbb{R}}\left(u^{2}+u_{x}^{2}\right)(t, \cdot) \Psi\left(\cdot-x\left(t_{0}\right)-\frac{\theta}{2}\left(t-t_{0}\right)+R\right) \leq \int_{\mathbb{R}}\left(u^{2}+u_{x}^{2}\right)\left(t_{0}, \cdot\right) \Psi\left(x-x\left(t_{0}\right)+R\right)+K_{0}(\alpha) e^{-R / 6}
$$


which leads to

$$
\begin{aligned}
\int_{\mathbb{R}}\left(\eta^{2}+\eta_{x}^{2}\right)(t, \cdot) & \Psi\left(\cdot-x\left(t_{0}\right)-\frac{\theta}{2}\left(t-t_{0}\right)+R\right)=\int_{\mathbb{R}}\left(u^{2}+u_{x}^{2}\right)(t, \cdot) \Psi\left(\cdot-x\left(t_{0}\right)-\frac{\theta}{2}\left(t-t_{0}\right)+x_{0}\right) \\
& -2 c_{0} \int_{\mathbb{R}}\left(u(t) \varphi(\cdot-x(t))+u_{x}(t) \varphi_{x}(\cdot-x(t)) \Psi\left(\cdot-x\left(t_{0}\right)-\frac{\theta}{2}\left(t-t_{0}\right)+R\right)\right. \\
& +c_{0}^{2} \int_{\mathbb{R}}\left(\varphi^{2}+\varphi_{x}^{2}\right)(t, \cdot-x(t)) \Psi\left(\cdot-x\left(t_{0}\right)-\frac{\theta}{2}\left(t-t_{0}\right)+R\right) \\
& \leq \int_{\mathbb{R}}\left(u^{2}+u_{x}^{2}\right)\left(t_{0}, \cdot\right) \Psi\left(\cdot-x\left(t_{0}\right)+R\right)+K_{0}(\alpha) e^{-R / 6} \\
& -2 c_{0} \int_{\mathbb{R}}\left(u\left(t_{0}\right) \varphi\left(\cdot-x\left(t_{0}\right)\right)+u_{x}\left(t_{0}\right) \varphi_{x}\left(\cdot-x\left(t_{0}\right)\right) \Psi\left(\cdot-x\left(t_{0}\right)+R\right)+C \delta\right. \\
& +c_{0}^{2} \int_{\mathbb{R}}\left(\varphi^{2}+\varphi_{x}^{2}\right)\left(t_{0}, \cdot-x\left(t_{0}\right)\right) \Psi\left(\cdot-x\left(t_{0}\right)+R\right)+C e^{-R / 6} \\
& \lesssim \int_{\mathbb{R}}\left(\eta^{2}+\eta_{x}^{2}\right)(t, \cdot) \Psi\left(\cdot-x\left(t_{0}\right)+R\right)+C\left(e^{-R / 6}+\delta\right) \\
& \lesssim \delta+e^{-R / 6}
\end{aligned}
$$

where in the next to the last step we used that $\varphi$ decays exponentially fast and (5.25) since $x(t)-x\left(t_{0}\right)-\frac{\theta}{2}\left(t-t_{0}\right)+R \geq R$ for all $t \geq t_{0}$. Taking $R$ large enough and $t_{1}>t_{0}$ such that $\theta t_{1} \geq x\left(t_{0}\right)+\frac{\theta}{2}\left(t_{1}-t_{0}\right)-R$, it follows that for $t \geq t_{1}$,

$$
\int_{\mathbb{R}}\left(\eta^{2}+\eta_{x}^{2}\right)(t, \cdot) \Psi(\cdot-\alpha t) \lesssim \delta
$$

which completes the proof of Theorem 1.2 with $c^{*}=c_{0}$.

\section{Asymptotic STABiLity of tRAin OF PEAKOnS}

In [21] the orbital stability in $H^{1}(\mathbb{R})$ of well ordered trains of peakons is established. More precisely, the following theorem is proved ${ }^{6}$ :

Theorem 6.1 ([21]). Let be given $N$ velocities $c_{1}, . ., c_{N}$ such that $0<c_{1}<c_{2}<$ ..$<c_{N}$. There exist $n_{0} \in \mathbb{N}$ satisfying (5.6), $A>0, L_{0}>0$ and $\varepsilon_{0}>0$ such that if $u \in C\left(\mathbb{R} ; H^{1}\right)$ is the global solution of $(C-H)$ emanating from $u_{0} \in Y_{+}$, with

$$
\left\|u_{0}-\sum_{j=1}^{N} \varphi_{c_{j}}\left(\cdot-z_{j}^{0}\right)\right\|_{H^{1}} \leq \varepsilon^{2}
$$

for some $0<\varepsilon<\varepsilon_{0}$ and $z_{j}^{0}-z_{j-1}^{0} \geq L$, with $L>L_{0}$, then there exist $N C^{1}$ functions $t \mapsto x_{1}(t), . ., t \mapsto x_{N}(t)$ uniquely determined such that

$$
\sup _{t \in \mathbb{R}+}\left\|u(t, \cdot)-\sum_{j=1}^{N} \varphi_{c_{j}}\left(\cdot-x_{j}(t)\right)\right\|_{H^{1}} \leq A \sqrt{\sqrt{\varepsilon}+L^{-1 / 8}}
$$

\footnotetext{
${ }^{6}$ Here again, in the statement given in [21], $\partial_{x} \varphi_{c_{i}}$ appears instead of $\rho_{n_{0}} * \partial_{x} \varphi_{c_{i}}$ in the orthogonality condition (6.3) and thus there is a gap in the proof of the $C^{1}$-regularity of the functions $x_{i}, i=1, . ., N$. The modifications to get the statement below are exactly the same as the ones to get Lemma 5.1 that is proven in the appendix.
} 
and

$$
\int_{\mathbb{R}}\left(u(t, \cdot)-\sum_{j=1}^{N} \varphi_{c_{j}}\left(\cdot-x_{j}(t)\right)\right)\left(\rho_{n_{0}} * \partial_{x} \varphi_{c_{i}}\right)\left(\cdot-x_{i}(t)\right) d x=0, \quad i \in\{1, \ldots, N\} .
$$

Moreover, for $i=1, . ., N$

$$
\left|\dot{x}_{i}-c_{i}\right| \leq A \sqrt{\sqrt{\varepsilon}+L^{-1 / 8}}, \quad \forall t \in \mathbb{R}_{+} .
$$

Combining this result with the asymptotic stability of a peakon established in the preceding section, we are able to extend the asymptotic result to a train of well ordered peakons by following the strategy developped in [28] (see also [20]).

Theorem 6.2. Let be given $N$ velocities $c_{1}, . ., c_{N}$ such that $0<c_{1}<c_{2}<. .<c_{N}$ and $0<\theta_{0}<c_{1} / 4$. There exist $L_{0}>0$ and $\varepsilon_{0}>0$ such that if $u \in C\left(\mathbb{R} ; H^{1}\right)$ is the solution of $(C-H)$ emanating from $u_{0} \in Y_{+}$, with

$$
\left\|u_{0}-\sum_{j=1}^{N} \varphi_{c_{j}}\left(\cdot-z_{j}^{0}\right)\right\|_{H^{1}} \leq \varepsilon_{0}^{2} \quad \text { and } \quad z_{j}^{0}-z_{j-1}^{0} \geq L_{0},
$$

then there exist $0<c_{1}^{*}<. .<c_{N}^{*}$ and $C^{1}$-functions $t \mapsto x_{1}(t), . ., t \mapsto x_{N}(t)$, with $\dot{x}_{j}(t) \rightarrow c_{j}^{*}$ as $t \rightarrow+\infty$, such that,

$$
u\left(\cdot+x_{j}(t)\right) \underset{t \rightarrow+\infty}{\rightarrow} \varphi_{c_{j}^{*}} \text { in } H^{1}(\mathbb{R}), \forall j \in\{1, . ., N\}
$$

Moreover,

$$
u-\sum_{j=1}^{N} \varphi_{c_{j}^{*}}\left(\cdot-x_{j}(t)\right) \underset{t \rightarrow+\infty}{\longrightarrow} 0 \text { in } H^{1}(] \theta_{0} t,+\infty[) .
$$

Finally, we will make use of the fact that Camassa-Holm equation possesses special solutions called multipeakons given by

$$
u(t, x)=\sum_{i=1}^{N} p_{i}(t) e^{-\left|x-q_{i}(t)\right|}
$$

where $\left(p_{i}(t), q_{i}(t)\right), i=1, . ., N$, satisfy a differential Hamiltonian system (cf. [9]). In [2] (see also [9]), the limits as $t \rightarrow \mp \infty$ of $p_{i}(t)$ and $\dot{q}_{i}(t), i=1, . ., N$, are determined. Combining the orbital stability of well ordered train of peakons, the continuity with respect to initial data in $H^{1}(\mathbb{R})$ and the asymptotics of multipeakons, the $H^{1}$-stability of the variety

$$
\mathcal{N}:=\left\{v=\sum_{i=1}^{N} p_{j} e^{-\left|\cdot-q_{j}\right|},\left(p_{1}, . ., p_{N}\right) \in\left(\mathbb{R}_{+}\right)^{N}, q_{1}<q_{2}<. .<q_{N}\right\} .
$$

is proved in ([21], Corollary 1.1). Gathering this last result with the asymptotics of the multipeakons and Theorem 6.2, the following asymptotic stability result for not well ordered train of peakons can be deduced quite directly.

Corollary 6.1. Let be given $N$ positive real numbers $p_{1}^{0}, . ., p_{N}^{0}, N$ real numbers $q_{1}^{0}<. .<q_{N}^{0}$ and let $0<\lambda_{1}<. .<\lambda_{N}$ be the $N$ distinct eigenvalues of the matrix $\left(p_{j}^{0} e^{-\left|q_{i}^{0}-q_{j}^{0}\right| / 2}\right)_{1 \leq i, j \leq N}$. For any $B>0$ there exists a positive function $\varepsilon$ with $\varepsilon(y) \rightarrow$ 
0 as $y \rightarrow 0$ and $\alpha_{0}>0$ such that if $u_{0} \in H^{1}(\mathbb{R})$ satisfies $m_{0}:=u_{0}-u_{0, x x} \in \mathcal{M}_{+}(\mathbb{R})$ with

$$
\left\|m_{0}\right\|_{\mathcal{M}} \leq B \quad \text { and } \quad\left\|u_{0}-\sum_{j=1}^{N} p_{j}^{0} \exp \left(\cdot-q_{j}^{0}\right)\right\|_{H^{1}} \leq \alpha
$$

for some $0<\alpha<\alpha_{0}$, then there exists $0<c_{1}^{*}<\cdot \cdot<c_{N}^{*}$ and $C^{1}$-functions $\left(x_{1}, . ., x_{N}\right)$ with

$$
\left|c_{i}^{*}-\lambda_{i}\right| \leq \varepsilon(\alpha) \quad \text { and } \quad \lim _{t \rightarrow+\infty} \dot{x}_{i}(t)=c_{i}^{*}, \quad \forall i \in\{1, . ., N\}
$$

such that

$$
u-\sum_{i=1}^{N} \varphi_{c_{i}^{*}}\left(\cdot-x_{i}(t)\right) \underset{t \rightarrow+\infty}{\longrightarrow} 0 \text { in } H^{1}(] \frac{\lambda_{1} t}{4},+\infty[)
$$

Proof of Theorem 6.2. We first concentrate ourself on the fastest bump located around $x_{N}(\cdot)$. To adapt Lemma 3.2 to this bump, we define $I_{t_{0}}^{N, x_{0}}(\cdot)$ as $I_{t_{0}}^{x_{0}}(\cdot)$ in Lemma 3.2 but with $z_{t_{0}}^{x_{0}}(\cdot)$ replaced by

$$
z_{t_{0}}^{N, x_{0}}=x_{N}\left(t_{0}\right)+x_{0}+z(t)-z\left(t_{0}\right)
$$

where $z: \mathbb{R} \rightarrow \mathbb{R}$ is a $C^{1}$-function that satisfies

$$
(1-\alpha) \dot{x}_{N}(t) \leq \dot{z}(t) \leq(1-\beta) \dot{x}_{N}(t)
$$

for some $0<\beta \leq \alpha<1$. We start by noticing that to prove (3.8) we can replace (3.5) by the less restrictive condition :

$$
\|u(t)\|_{L^{\infty}\left(x-x(t)>R_{0}\right)} \leq \frac{(1-\alpha) c_{0}}{2^{6}} .
$$

Indeed, it is direct to check that the condition $x-x(t) \leq R_{0}$ is sufficient to get (3.17). Therefore, to get the same estimate as (3.8) for $I_{t_{0}}^{N, \bar{R}}(\cdot)$ it suffices to assume that there exists $R_{0}>0$ and $0<\alpha<1$ such that

$$
\|u(t)\|_{L^{\infty}\left(x-x_{N}(t)>R_{0}\right)} \leq \frac{(1-\alpha) c_{N}}{2^{6}}, \forall t \in \mathbb{R} .
$$

Now, because of the presence of the bumps at the left of the Nth bump, we are able to establish the same estimate as (3.9) only for $t_{0} \geq t_{R}$ where $t_{R}$ depends on $R$. To prove such version of (3.9) with $\alpha=\frac{5}{8} \frac{c_{N}-c_{N-1}}{c_{N}}$ we replace (3.5) by

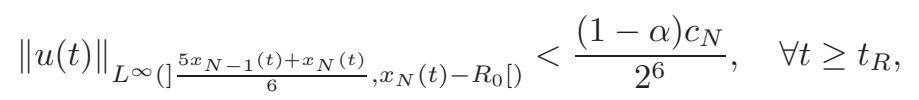

with $x_{N}(t)-x_{N-1}(t) \geq 2 R$. For any $R \geq R_{0}$ we define

$$
t_{R}=0 \vee\left\{t \geq 0, x_{N}(t)-x_{N-1}(t)=2 R\right\} .
$$

For $R \geq R_{0}$, assuming that $z(\cdot)$ satisfies $(6.10)$ and that

$$
\left|c_{N}-\dot{x}_{N}(t)\right|+\left|c_{N-1}-\dot{x}_{N-1}(t)\right| \leq \frac{1}{12}\left(c_{N}-c_{N-1}\right), \forall t \geq 0,
$$


we get for $x \leq \frac{5 x_{N-1}(t)+x_{N}(t)}{6}$ and $t_{0} \geq t_{R}$,

$$
\begin{aligned}
x-z_{t_{0}}^{N,-R} & =x-x_{N}(t)+R+(x(t)-z(t))-\left(x\left(t_{0}\right)-z\left(t_{0}\right)\right) \\
& \leq-\frac{5}{6}\left(x_{N}(t)-x_{N-1}(t)\right)+R+\alpha c_{N}\left(t-t_{0}\right) \\
& \leq-\frac{5}{3} R-\frac{3}{4}\left(c_{N}-c_{N-1}\right)\left(t-t_{0}\right)+R+\frac{5}{8}\left(c_{N}-c_{N-1}\right)\left(t-t_{0}\right) \\
& \leq-\frac{2}{3} R-\frac{1}{8}\left(c_{N}-c_{N-1}\right)\left(t-t_{0}\right),
\end{aligned}
$$

where we took $\alpha=\frac{5}{8} \frac{c_{N}-c_{N-1}}{c_{N}}$. This leads to

$$
\Psi\left(x-z_{t_{0}}^{N,-R}\right) \lesssim e^{-\frac{R}{9}} e^{-\frac{1}{48}\left(c_{N}-c_{N-1}\right)\left(t-t_{0}\right)} .
$$

which is sufficient to get $(3.18)$ with $\beta c_{0}$ replaced by $\frac{c_{N}-c_{N-1}}{48}$.

In the sequel, we set

$$
\varepsilon_{0}=\left(\frac{\sigma_{0}}{2^{18}}\right)^{8} \text { and } L_{0}=\left(\frac{\sigma_{0}}{2^{18}}\right)^{16} \quad \text { where } \sigma_{0}=A\left(\min _{i=2, . ., N}\left(c_{i}-c_{i-1}\right) \wedge \theta\right)
$$

Taking $\alpha=\frac{5}{8} \frac{c_{N}-c_{N}-1}{c_{N}}$, we infer from (6.2) that for $R_{0}$ such that

$$
N C_{N} e^{-R_{0}}<\frac{\sigma_{0}}{2^{18}}
$$

(6.11) is satisfied . Moreover, for $R \geq R_{0},(6.4)$ ensures (6.14) is satisfied and that (6.12) is satisfied with $t_{R}$ defined as in (6.13). Therefore, taking $z(\cdot)=(1-\alpha) x_{N}(\cdot)$ and $\beta=\alpha=\frac{5\left(c_{N}-c_{N-1}\right)}{8 c_{N}}$, we infer that for any $R>R_{0}$

$$
I_{t_{0}}^{N,+R}\left(t_{0}\right)-I_{t_{0}}^{N,+R}(t) \leq K_{0} e^{-R / 6}, \quad \forall 0 \leq t \leq t_{0}
$$

and

$$
I_{t_{0}}^{N,-R}(t)-I_{t_{0}}^{N,-R}\left(t_{0}\right) \leq K_{0} e^{-\frac{R}{24}}, \quad \forall t \geq t_{0} \geq t_{R}
$$

where $t_{R}$ is defined as in (6.13) and where $K_{0}=K_{0}\left(\sigma_{0}\right)$. As in Section 5, this ensures that

$$
J_{\gamma, r}^{R}\left(u\left(t_{0}, \cdot+x_{N}\left(t_{0}\right)\right)\right) \leq J_{\gamma, r}^{R}\left(u\left(t, \cdot+x_{N}(t)\right)\right)+K_{0} e^{-R / 6}, \quad \forall 0 \leq t \leq t_{0},
$$

and

$$
J_{\gamma, l}^{R}\left(u\left(t_{0}, \cdot+x_{N}\left(t_{0}\right)\right)\right) \geq J_{\gamma, l}^{R}\left(u\left(t, \cdot+x_{N}(t)\right)\right)-K_{0} e^{-\frac{R}{24}}, \quad \forall t \geq t_{0} \geq t_{R} .
$$

Since, we only need these last two estimates in the proof of Proposition 5.2 as well as in Subsections 5.1-5.3, we infer that there exists $c_{N}^{*}$ close to $c_{N}$ such that $\dot{x}_{N} \rightarrow c_{N}^{*}$ as $t \rightarrow+\infty$ and

$$
u\left(t, \cdot+x_{N}(t)\right) \underset{t \rightarrow+\infty}{\rightarrow} c_{N}^{*} \varphi \text { in } H^{1}(\mathbb{R}) .
$$

Moreover,

$$
u\left(t, \cdot+x_{N}(t)\right)-c_{N}^{*} \varphi \underset{t \rightarrow+\infty}{\longrightarrow} 0 \text { in } H^{1}(]-A,+\infty[) \text { for any } A>0 .
$$

Now, setting $y_{N}=\frac{x_{N}+x_{N-1}}{2}$ and noticing that for all $t \geq 0, z(\cdot)=y_{N}(\cdot)$ also satisfies (3.6) with $\alpha=\frac{5\left(c_{N}-c_{N-1}\right)}{8 c_{N}}$ and $\beta=\frac{\left(c_{N}-c_{N-1}\right)}{8 c_{N}}$, we get that for $R \geq R_{0}$,

$$
\int_{\mathbb{R}}\left(u^{2}+u_{x}^{2}\right)(t, \cdot) \Psi\left(\cdot-y_{N}(t)\right)=I_{t_{R}}^{N, y_{N}\left(t_{R}\right)-x_{N}\left(t_{R}\right)}(t)
$$


is also almost non increasing for $t \geq t_{R}$ where $t_{R}$ is defined in (6.13). Indeed, we have $x_{N}\left(t_{R}\right)-y_{N}\left(t_{R}\right) \geq R \geq R_{0}$.

This enables, as in Subsection 5.3, to prove that actually

$$
\int_{\mathbb{R}}\left[\left(u(t)-c_{N}^{*} \varphi\left(\cdot-x_{N}(t)\right)\right)^{2}+\left(u_{x}-c_{N}^{*} \varphi_{x}\left(\cdot-x_{N}(t)\right)\right)^{2}\right] \Psi\left(\cdot-y_{N}(t)\right) \underset{t \rightarrow \infty}{\longrightarrow} 0 .
$$

Let us now set $y_{i}=\left(x_{i}+x_{i-1}\right) / 2$ for $i=2, . ., N-1$ and $y_{1}(t)=\theta t$. We claim that if for some $1 \leq i \leq N-1$ it holds

$$
\int_{\mathbb{R}}\left[\left(u(t)-\sum_{j=i+1}^{N} c_{j}^{*} \varphi\left(\cdot-x_{N}(t)\right)\right)^{2}+\left(u_{x}-\sum_{j=i+1}^{N} c_{j}^{*} \varphi_{x}\left(\cdot-x_{N}(t)\right)\right)^{2}\right] \Psi\left(\cdot-y_{i+1}(t)\right) \underset{t \rightarrow \infty}{\longrightarrow} 0
$$

then $\dot{x}_{i}(t) \rightarrow c_{i}^{*}$ as $t \rightarrow \infty$ for some $c_{i}^{*}$ close to $c_{i}$ and

$$
\int_{\mathbb{R}}\left[\left(u(t)-\sum_{j=i}^{N} c_{j}^{*} \varphi\left(\cdot-x_{N}(t)\right)\right)^{2}+\left(u_{x}-\sum_{j=i}^{N} c_{j}^{*} \varphi_{x}\left(\cdot-x_{N}(t)\right)\right)^{2}\right] \Psi\left(\cdot-y_{i}(t)\right) \underset{t \rightarrow \infty}{\longrightarrow} 0
$$

which clearly yields the desired result by a finite iterative argument.

We start by noticing that for $i \in\{2, . ., N-1\},(6.12)$ also holds for $u$ with $\alpha=\frac{5\left(c_{i}-c_{i-1}\right)}{8 c_{i}}$ and with $x_{N}, x_{N-1}, c_{N}, c_{N-1}$ and $t_{R}$ replaced by respectively $x_{i}$, $x_{i-1}, c_{i}$ and $c_{i-1}$ and

$$
t_{R}^{i}=\max \left(\{0\} \cup\left\{t \geq 0, x_{i}(t)-x_{i-1}(t)=2 R\right\}\right) .
$$

Moreover, for $i=1,(6.15)$ and (6.2) ensure that

$$
\|u(t)\|_{L^{\infty}(]-\infty, x_{1}(t)-R_{0}[}<\frac{1-\alpha}{2^{6}} c_{1}
$$

with $1-\alpha=\frac{\theta}{4 c_{1}}$. Therefore, defining $I_{t_{0}}^{i, x_{0}}$ in the same way as $I_{t_{0}}^{x_{0}}$ but with $x(\cdot)$ replaced by $x_{i}(\cdot)$ and taking $z(\cdot)=(1-\alpha) x_{i}(\cdot)$ with $\alpha=\beta=\frac{5\left(c_{i}-c_{i-1}\right)}{8 c_{i}}$ if $i \geq 2$ and $z(t)=\frac{\theta}{2} t, \alpha=1-\frac{\theta}{4 c_{1}}, \beta=1 / 4$ if $i=1$, we get that for any $R \geq R_{0}$ it holds

$$
I_{t_{0}}^{i,-R}(t)-I_{t_{0}}^{i,-R}\left(t_{0}\right) \leq K_{0} e^{-\frac{R}{24}}, \quad \forall i \in\{1, . ., N-1\}, \forall t \geq t_{0} \geq t_{R}^{i},
$$

where $K_{0}=K_{0}\left(\sigma_{0}\right)$. As in Section 5, it follows that for $\gamma \geq 0$ small enough,

$$
J_{\gamma, l}^{R}\left(u\left(t, \cdot+x_{i}(t)\right)\right) \geq J_{\gamma, l}^{R}\left(u\left(t_{0}, \cdot+x_{i}\left(t_{0}\right)\right)\right)-K_{0} e^{-\frac{R}{24}}, \forall t \geq t_{0} \geq t_{R}^{i} .
$$

Now, the proof of the almost monotonicity of $J_{\gamma, r}^{R}\left(u\left(t, \cdot+x_{i}(t)\right)\right)$ is more subtle. Indeed, starting at $x_{i}\left(t_{0}\right)+R$ at time $t_{0}$ for some $R>0$ and traveling back in time with a fixed speed strictly less than $c_{i}$, one will cross $x_{i+1}(\cdot)$ at some time $t$ which will tend to $+\infty$ if $t_{0}$ tends to $+\infty$. This is clearly not allowed if we want to prove an almost monotonicity result. To overcome this difficulty we will decompose the travel back into two parts. First, one travels back with some speed strictly less than $c_{i}$ till one crosses the curve of the middle point $y_{i+1}(\cdot)=\left(x_{i}(\cdot)+x_{i+1}(\cdot)\right) / 2$. Then, one continues to travel back but along $y_{i+1}(\cdot)$ until the time $t_{R}^{i+1}$ that satisfies $x_{i+1}\left(t_{R}^{i+1}\right)-x_{i}\left(t_{R}^{i+1}\right) \geq 2 R$. This is the idea of the proof of the following lemma which ensures that $J_{\gamma, r}^{R}\left(u\left(t, \cdot+x_{i}(t)\right)\right)$ is almost non increasing for $t \geq t_{R}^{i+1}$. 
Lemma 6.3. Let $i \in\{1, \ldots, N-1\}$ and $0 \leq \gamma \leq c_{i}$. For any $R>0$ it holds

$$
J_{\gamma, r}^{R}\left(u\left(t_{0}, \cdot+x_{i}\left(t_{0}\right)\right)\right) \leq J_{\gamma, r}^{R}\left(u\left(t, \cdot+x_{i}(t)\right)\right)+K_{0} e^{-\frac{R}{24}}, \forall t_{0} \geq t \geq t_{R}^{i+1} .
$$

where $t_{R}^{i}$ is defined as in (6.23).

Proof. Let $R>0$ and $t_{0}>t_{R}^{i}$. We set

$$
t_{0}^{\prime}=t_{R}^{i+1} \vee\left\{t \in\left[t_{R}^{i+1}, t_{0}\right], x_{i}\left(t_{0}\right)+R+\frac{3}{4}\left(x_{i}(t)-x_{i}\left(t_{0}\right)\right)=y_{i+1}(t)\right\}
$$

On $\left[t_{0}^{\prime}, t_{0}\right]$ it holds

$$
x_{i}\left(t_{0}\right)+R+\frac{3}{4}\left(x_{i}(t)-x_{i}\left(t_{0}\right)\right) \leq y_{i+1}(t)
$$

and thus seting $z_{t_{0}}^{i, R}(t)=x_{i}\left(t_{0}\right)+R+\frac{3}{4}\left(x_{i}(t)-x_{i}\left(t_{0}\right)\right)$, we get for any $t \geq t_{R}^{i+1}$ and any $x \geq \frac{5 x_{i+1}(t)+3 x_{i}(t)}{8}$ that

$$
x-z_{t_{0}}^{i, R}(t) \geq x-y_{i+1}(t) \geq \frac{R}{4}+\frac{1}{2^{4}}\left(c_{i+1}-c_{i}\right)\left(t-t_{R}^{i+1}\right)
$$

which leads to

$$
\Psi\left(x-z_{t_{0}}^{i, R}(t)\right) \leq e^{-\frac{R}{24}} e^{-\frac{1}{2^{7}}\left(c_{i+1}-c_{i}\right)\left(t-t_{R}^{i}\right)} .
$$

On the other, (6.15) and (6.2) ensure that for $R \geq R_{0}$ (with $R_{0}$ defined in (6.16)),

$$
\|u(t)\|_{L^{\infty}(] x_{i}(t)+R, \frac{5 x_{i+1}(t)+3 x_{i}(t)}{8}[}<\frac{\theta}{2^{8}}<\frac{1}{2} \frac{c_{i}}{2^{6}}, \quad \forall t \geq t_{R}^{i+1},
$$

Therefore, defining

$$
I_{t_{0}}^{i, R}(t)=\left\langle u^{2}(t)+u_{x}^{2}(t)+\gamma y(t), \Psi\left(\cdot-z_{t_{0}}^{i, R}(t)\right)\right\rangle,
$$

with $0 \leq \gamma \leq c_{i}$, we get as in (3.8) that for all $t \in\left[t_{0}^{\prime}, t_{0}\right]$,

$$
I_{t_{0}}^{i, R}\left(t_{0}\right)-I_{t_{0}}^{i, R}(t) \leq K_{0} e^{-\frac{R}{24}} .
$$

It follows as in (5.14) that

$$
J_{\gamma, r}^{R}\left(u\left(t, \cdot+x_{i}\left(t_{0}\right)\right) \leq J_{\gamma, r}^{R}\left(u\left(t, \cdot+x_{i}(t)\right)+K_{0} e^{-\frac{R}{24}}, \quad \forall t \in\left[t_{0}^{\prime}, t_{0}\right]\right.\right.
$$

If $t_{0}^{\prime}=t_{R}^{i+1}$ we are done. Otherwise we must have $z_{t_{0}^{\prime}}^{i, R}=y_{i+1}\left(t_{0}^{\prime}\right)$. But then the same arguments as in (6.20) lead, for $0 \leq \gamma \leq c_{i}$, to

$$
\left\langle u^{2}(t)+u_{x}^{2}(t)+\gamma y(t), \Psi\left(\cdot-y_{i+1}\left(t_{0}^{\prime}\right)\right)\right\rangle \leq\left\langle u^{2}(t)+u_{x}^{2}(t)+\gamma y(t), \Psi\left(\cdot-y_{i+1}(t)\right)\right\rangle+K_{0} e^{-R / 9}, \forall t \in\left[t_{R}^{i+1}, t_{0}^{\prime}\right] .
$$

Since for $t \geq t_{R}^{i+1}$,

$$
\left\langle u^{2}(t)+u_{x}^{2}(t)+\gamma y(t), \Psi\left(\cdot-y_{i+1}(t)\right)\right\rangle \leq J_{\gamma, r}^{R}\left(u\left(t, \cdot+x_{i}(t)\right)\right.
$$

we obtain the desired result for all $t \in\left[t_{R}^{i+1}, t_{0}\right]$.

Since the proof of Proposition 5.2 only uses the almost monotonicity of $J_{\gamma, r}^{R}(u(t, \cdot+$ $x(t)))$ and $J_{\gamma, l}^{R}(u(t, \cdot+x(t)))$ for some $\gamma>0$ and for $t \geq t_{R}$ where $t_{R}$ is a non negative time depending on $R$, we obtain as in (5.18) that

$$
u\left(t, \cdot+x_{i}(t)\right)-\lambda_{i}(t) \varphi \underset{t \rightarrow+\infty}{\longrightarrow} 0 \text { in } H_{l o c}^{1}(\mathbb{R}) .
$$

where

$$
\lambda_{i}(t)=\max _{x \in\left[-c_{i}, c_{i}\right]} u\left(t, x+x_{i}(t)\right) .
$$


Let us now set

$$
W_{>i}(t)=\sum_{j=i+1}^{N} c_{j}^{*} \varphi\left(\cdot-x_{j}(t)\right) \text { and } v=u-W_{>i}(t),
$$

In view of (6.4), for any $\varepsilon>0$, there exists $t_{\varepsilon}>0$ such that for all $R \geq 0$ and $t \geq t_{\varepsilon}$,

$$
\mid J_{0, l}^{R}\left(u\left(t, \cdot+x_{i}(t)\right)-J_{0, l}^{R}\left(v\left(t, \cdot+x_{i}(t)\right) \mid \leq \varepsilon .\right.\right.
$$

Moreover, decomposing $J_{0, r}^{R}\left(u\left(t, \cdot+x_{i}(t)\right)\right)$ as

$$
\begin{aligned}
J_{0, r}^{R}\left(u\left(t, \cdot+x_{i}(t)\right)\right) & =\int_{\mathbb{R}}\left(u^{2}+u_{x}^{2}\right) \Psi\left(\cdot-x_{i}(t)-R\right)\left[1-\Psi\left(\cdot-y_{i+1}(t)\right)\right] \\
& +\int_{\mathbb{R}}\left(u^{2}+u_{x}^{2}\right) \Psi\left(\cdot-x_{i+1}(t)-R\right) \Psi\left(\cdot-y_{i+1}(t)\right) \\
& =A_{1}(t)+A_{2}(t),
\end{aligned}
$$

(6.4) ensures that

$$
A_{1}(t)-\int_{\mathbb{R}}\left(v^{2}+v_{x}^{2}\right) \Psi\left(\cdot-x_{i}(t)-R\right)\left[1-\Psi\left(\cdot-y_{i+1}(t)\right)\right] \underset{t \rightarrow \infty}{\longrightarrow} 0
$$

and (6.21) together with (6.4) ensures that

$$
A_{2}(t)-\int_{\mathbb{R}}\left(v^{2}+v_{x}^{2}\right) \Psi\left(\cdot-x_{i}(t)-R\right) \Psi\left(\cdot-y_{i+1}(t)\right) \underset{t \rightarrow \infty}{\longrightarrow} \sum_{i+1}^{N} E\left(\varphi_{c_{i}^{*}}\right) .
$$

This proves that $J_{0, r}^{R}\left(v\left(t, \cdot+x_{i}(t)\right)\right)$ and $J_{0, l}^{R}\left(v\left(t, \cdot+x_{i}(t)\right)\right)$ enjoy the same almost monotonicity property as respectively $J_{0, r}^{R}\left(v\left(t, \cdot+x_{i}(t)\right)\right)$ and $J_{0, l}^{R}\left(v\left(t, \cdot+x_{i}(t)\right)\right)$ for $t \geq t_{R}$ large enough.

Let now $\delta>0$ be fixed. According to (6.21), there exists $t_{\delta}>0$ such that

$$
\int_{\mathbb{R}}\left(v^{2}+v_{x}^{2}\right)\left(t_{\delta}, x\right) \Psi\left(x-y_{i+1}\left(t_{\delta}\right)\right) d x<\delta / 3 .
$$

Moreover, by (6.4), we may also require

$$
\int_{\mathbb{R}}\left(\varphi^{2}+\varphi_{x}^{2}\right)\left(x-x_{i}(t)\right) \Psi\left(x-y_{i+1}\left(t_{\delta}\right)\right) d x<\delta / 3 .
$$

Therefore using the almost monotonicity of $J_{0, r}^{R}\left(v\left(t, \cdot+x_{i}(t)\right)\right)$, with $R=y_{i+1}\left(t_{\delta}\right)-$ $x_{i}\left(t_{\delta}\right)$, together with the local strong convergence result (6.29) and (6.4), we get that for all fixed $A>0$,

$$
u\left(t, \cdot+x_{i}(t)\right)-\lambda_{i}(t) \varphi-W_{>i}\left(t, \cdot+x_{i}(t)\right) \underset{t \rightarrow+\infty}{\longrightarrow} 0 \text { in } H^{1}(]-A,+\infty[) .
$$

To prove the convergence of the scaling parameter $\lambda_{i}$ we use the above strong convergence in $H^{1}(]-A,+\infty[)$ and (6.4) to get that for any $\delta>0$ there exists $R_{\delta}>0$ and $t_{\delta}>0$ such that

$$
\left|\int_{\mathbb{R}}\left(v^{2}+v_{x}^{2}\right)(t, x) \Psi\left(x-x_{i}(t)+R_{\delta}\right) d x-\lambda_{i}^{2}(t) E(\varphi)\right| \leq \delta, \quad \forall t \geq t_{\delta} .
$$

The almost monotonicity of $J_{0, l}^{R_{\delta}}\left(v\left(t, \cdot+x_{i}(t)\right)\right)$ then ensures that $\lambda_{i}(t) \rightarrow c_{i}^{*}$ as $t \rightarrow+\infty$, for some $c_{i}^{*}$ close to $c_{i}$. Hence, we get

$$
u\left(t, \cdot+x_{i}(t)\right)-c_{i}^{*} \varphi-W_{>i}\left(t, \cdot+x_{i}(t)\right) \underset{t \rightarrow+\infty}{\longrightarrow} 0 \text { in } H^{1}(]-A,+\infty[) .
$$


To get the convergence of $\dot{x}_{i}$ towards $c_{i}^{*}$ we write the equation for

$$
\eta=u-\sum_{j=i}^{N} c_{j}^{*} \varphi\left(\cdot-x_{j}(t)\right)=u-W_{\geq i}=v-c_{i}^{*} \varphi\left(\cdot-x_{i}(t)\right)
$$

and proceed as in Subsection 5.3.

Finally to prove (6.22) we first notice that, proceeding as in Subsection 5.4, we obtain that for any fixed $\delta>0$, there exists $R>0$ and $t_{0} \geq 0$ such that

$$
\begin{gathered}
\left|\sum_{j \geq i}^{N} E\left(\varphi_{c_{j^{*}}}\right)-\int_{\mathbb{R}}\left(W_{\geq i}+\left(\partial_{x} W_{\geq i}\right)^{2}\right) \Psi\left(\cdot-x_{i}(t)+y\right)\right|<\delta, \quad \forall y \geq R, \forall t \geq t_{0}, \\
\left\|\left(\eta^{2}+\eta_{x}^{2}\right)\left(t, \cdot+x_{i}(t)\right)\right\|_{H^{1}(]-2 R,+\infty[)}<\delta, \quad \forall t \geq t_{0},
\end{gathered}
$$

and

$\left|\sum_{j \geq i}^{N} E\left(\varphi_{c_{j^{*}}}\right)-\int_{\mathbb{R}}\left(u\left(t, \cdot+x_{i}(t)\right) W_{\geq i}+u_{x}\left(, \cdot+x_{i}(t)\right) \partial_{x} W_{\geq i}\right) \Psi(\cdot+y)\right|<\delta, \quad \forall y \geq R, \forall t \geq t_{0}$.

Therefore, using the almost monotonicity of $t \mapsto I_{t_{0}}^{i,-R}(t)$ with $\gamma=0, z(t)=$ $\frac{1}{2}\left(x_{i-1}(t)+y_{i}(t)\right),(1-\alpha)=\frac{c_{i}+7 c_{i-1}}{8 c_{i}}$ if $i \geq 2$ and $z(t)=\frac{\theta}{2} t, \alpha=1-\frac{\theta}{4 c_{1}}$ and $\beta=1 / 4$ if $i=1$, and proceeding as in Subsection (5.4), we get that for $t \geq t_{0}$,

$$
\begin{aligned}
\int_{\mathbb{R}}\left(\eta^{2}+\eta_{x}^{2}\right)(t, \cdot) & \Psi\left(\cdot-z_{t_{0}}^{i,-R}(t)\right)=\int_{\mathbb{R}}\left(u^{2}+u_{x}^{2}\right)(t, \cdot) \Psi\left(\cdot-z_{t_{0}}^{i,-R}(t)\right) \\
& -2 c_{i}^{*} \int_{\mathbb{R}}\left(u(t) W_{\geq i}(t)+u_{x}(t) \partial_{x} W_{\geq i}(t)\right) \Psi\left(\cdot-z_{t_{0}}^{i,-R}(t)\right) \\
& +\left(c_{i}^{*}\right)^{2} \int_{\mathbb{R}}\left(W_{\geq i}^{2}+\left(\partial_{x} W_{\geq i}\right)^{2}\right)(t) \Psi\left(\cdot-z_{t_{0}}^{i,-R}(t)\right) \\
& \leq \int_{\mathbb{R}}\left(v^{2}+v_{x}^{2}\right)\left(t_{0}, \cdot\right) \Psi\left(\cdot-x_{i}\left(t_{0}\right)+R\right)+K_{0}\left(\sigma_{0}\right) e^{-R / 6} \\
& -2 c_{i}^{*} \int_{\mathbb{R}}\left(u\left(t_{0}\right) W_{\geq i}\left(t_{0}\right)+u_{x}\left(t_{0}\right) \partial_{x} W_{\geq i}\left(t_{0}\right)\right) \Psi\left(\cdot-x_{i}\left(t_{0}\right)+R\right) \\
& +\left(c_{i}^{*}\right)^{2} \int_{\mathbb{R}}\left(W_{\geq i}^{2}+\left(\partial_{x} W_{\geq i}\right)^{2}\right)\left(t_{0}\right) \Psi\left(\cdot-x_{i}\left(t_{0}\right)+R\right)+2 \delta \\
& \leq \int_{\mathbb{R}}\left(\eta^{2}+\eta_{x}^{2}\right)(t, \cdot) \Psi\left(\cdot-x_{i}\left(t_{0}\right)+R\right)+K_{0}\left(\sigma_{0}\right) e^{-R / 6}+2 \delta \\
& \lesssim \delta+e^{-R / 6}
\end{aligned}
$$

where $z_{t_{0}}^{i,-R}(t)=x_{i}\left(t_{0}\right)-R+z(t)-z\left(t_{0}\right) \leq x_{i}(t)-R, \forall t \geq t_{0}$. This yields the result since, $R$ being fixed, it holds $z_{t_{0}}^{i,-R}(t) \leq y_{i}(t)$ for $t$ large enough.

\section{Appendix}

7.1. Proof of (3.13). Using that

$$
\frac{d}{d t} \int_{\mathbb{R}} u^{2}+u_{x}^{2}=2 \int_{\mathbb{R}} u u_{t} g+2 \int_{\mathbb{R}} u_{x} u_{x t} g
$$


(1.5) yields

$$
\begin{aligned}
2 \int_{\mathbb{R}} u u_{t} g & =-2 \int_{\mathbb{R}} u^{2} u_{x} g-2 \int_{\mathbb{R}} u p_{x} *\left(u^{2}+u_{x}^{2} / 2\right) g \\
& =\frac{2}{3} \int_{\mathbb{R}} u^{3} g^{\prime}-2 \int_{\mathbb{R}} u p_{x} *\left(u^{2}+u_{x}^{2} / 2\right) g
\end{aligned}
$$

and, recalling that $p_{x x}=p-\delta_{0}$,

$$
\begin{aligned}
2 \int_{\mathbb{R}} u_{x} u_{x t} g= & -2 \int_{\mathbb{R}} u_{x}^{3} g-2 \int_{\mathbb{R}} u_{x} u u_{2 x} g-2 \int_{\mathbb{R}} u_{x} p_{x x} *\left(u^{2}+u_{x}^{2} / 2\right) g \\
= & -2 \int_{\mathbb{R}} u_{x}^{3} g+\int_{\mathbb{R}} u_{x}^{3} g+\int_{\mathbb{R}} u u_{x}^{2} g^{\prime} \\
& -2 \int_{\mathbb{R}} u_{x} p *\left(u^{2}+u_{x}^{2} / 2\right) g+2 \int_{\mathbb{R}} u_{x}\left(u^{2}+u_{x}^{2} / 2\right) g \\
= & \int_{\mathbb{R}} u u_{x}^{2} g^{\prime}+2 \int_{\mathbb{R}} u p *\left(u^{2}+u_{x}^{2} / 2\right) g^{\prime}+2 \int_{\mathbb{R}} u p_{x} *\left(u^{2}+u_{x}^{2} / 2\right) g-\frac{2}{3} \int_{\mathbb{R}} u^{3} g^{\prime} .
\end{aligned}
$$

Gathering (7.1)-(7.3), (3.13) follows.

7.2. Proof of Lemma 5.1. Let $n_{0} \in \mathbb{N}$ to be specified later. For $z \in \mathbb{R}$ we define the function

$$
\begin{aligned}
\mathbb{R} \times H^{1}(\mathbb{R}) & \longrightarrow \mathbb{R} \\
(y, v) & \mapsto \int_{\mathbb{R}} v(x)\left(\rho_{n_{0}} * \varphi\right)^{\prime}(x-z-y) d x
\end{aligned}
$$

Since $\rho_{n_{0}}$ and $\varphi$ are both even, one has $Y_{z}(0, \varphi(\cdot-z))=0$. Moreover, $Y$ is clearly of class $C^{1}$ and it holds

$$
\frac{\partial Y_{z}}{\partial y}(0, \varphi(\cdot-z))=\int_{\mathbb{R}} \varphi^{\prime}\left(\rho_{n_{0}} * \varphi^{\prime}\right)=\left\|\varphi^{\prime}\right\|_{L^{2}}^{2}-\varepsilon\left(n_{0}\right)=1-\varepsilon\left(n_{0}\right),
$$

with $\varepsilon(n) \rightarrow 0$ as $n \rightarrow+\infty$. Therefore by taking $n_{0}$ large enough, we may require that

$$
\frac{\partial Y_{z}}{\partial y}(0, \varphi(\cdot-z)) \geq 1 / 2 .
$$

From the implicit function theorem we deduce that there exists $\tilde{\varepsilon}_{0}>0, \kappa_{0}>0$ and a $C^{1}$-function $y_{z}$ from $B_{H^{1}}\left(\varphi(\cdot-z), \tilde{\varepsilon}_{0}\right)$ in $] \kappa_{0}, \kappa_{0}[$ which is uniquely determined such that

$$
Y_{z}\left(y_{z}(u), u\right)=Y(0, \varphi)=0, \quad \forall u \in B_{H^{1}}\left(\varphi(\cdot-z), \tilde{\varepsilon}_{0}\right) .
$$

In particular there exists $C_{0}>0$ such that if $u \in B_{H^{1}}(\varphi(\cdot-z), \beta)$ with $0<\beta \leq \tilde{\varepsilon}_{0}$ then

$$
\left|y_{z}(u)\right| \leq C_{0} \beta \text {. }
$$

Note that, by a translation symmetry argument, $\tilde{\varepsilon}_{0}, \kappa_{0}$ and $C_{0}$ are independent of $z \in \mathbb{R}$. Therefore, by uniqueness, we can define a $C^{1}$-mapping $\tilde{x}$ from $U_{z \in \mathbb{R}} B_{H^{1}}\left(\varphi(\cdot-z), \tilde{\varepsilon}_{0}\right)$ into $] \kappa_{0}, \kappa_{0}$ [ by setting

$$
\tilde{x}(u)=z+y_{z}(u) \text { for } u \in B_{H^{1}}\left(\varphi(\cdot-z), \tilde{\varepsilon}_{0}\right) .
$$

Now we notice that $Y_{z}$ is also a $C^{1}$-function from $\mathbb{R} \times L^{2}(\mathbb{R})$ into $\mathbb{R}$ with

$$
\frac{\partial Y_{z}}{\partial y}(y, \varphi(\cdot-z))=\int_{\mathbb{R}} u(x)\left(\rho_{n_{0}}^{\prime \prime} * \varphi\right)(x-z-y) d x .
$$


Therefore, in the same way as above we obtain that there exists $\tilde{\tilde{\varepsilon}}_{0}>0$ and a $C^{1}$-function $\tilde{\tilde{x}}$ from $\cup_{z \in \mathbb{R}} B_{L^{2}}\left(\varphi(\cdot-z), \tilde{\tilde{\varepsilon}}_{0}\right)$ into a neighborhood of 0 in $\mathbb{R}$ such that

$$
\int_{\mathbb{R}} u\left(\rho_{n_{0}} * \varphi\right)^{\prime}(\cdot-y)=0 \Leftrightarrow y=\tilde{\tilde{x}}(u), \quad \forall u \in \cup_{z \in \mathbb{R}} B_{L^{2}}\left(\varphi(\cdot-z), \tilde{\tilde{\varepsilon}}_{0}\right) .
$$

We set $\varepsilon_{0}=\tilde{\varepsilon}_{0} \wedge \tilde{\tilde{\varepsilon}}_{0}$. By uniqueness it holds $\tilde{\tilde{x}} \equiv \tilde{x}$ on $B_{H^{1}}\left(\varphi(\cdot-z), \varepsilon_{0}\right)$ and thus $\tilde{x}$ is also a $C^{1}$-function on $\cup_{z \in \mathbb{R}} B_{H^{1}}\left(\varphi(\cdot-z), \varepsilon_{0}\right)$ equipped with the metric inducted by the $L^{2}(\mathbb{R})$-metric.

Now, according to (5.3), it holds $\left\{\frac{1}{c} u(t),, t \in \mathbb{R}\right\} \subset \cup_{z \in \mathbb{R}} B_{H^{1}}\left(\varphi(\cdot-z), \varepsilon_{0}\right)$ so that we can define the function $x(\cdot)$ on $\mathbb{R}$ by setting $x(t)=\tilde{x}(u(t))$. By construction $x(\cdot)$ satisfies (5.4)-(5.5). Moreover, (5.8) together with (7.5) ensure that for any $c>0$ and any $0<\varepsilon<c \varepsilon_{0}$, it holds

$$
\left.\left\|\frac{1}{c} u(t)-\varphi(\cdot-x(t))\right\|_{H^{1}} \leq\left(\frac{\varepsilon}{c}\right)^{2}+\sup _{|z| \leq C_{0}\left(\frac{\varepsilon}{c}\right)^{2}} \| \varphi-\varphi(\cdot-z)\right) \|_{H^{1}} \lesssim\left(\frac{\varepsilon}{c}\right)^{2}+\sqrt{C_{0}} \frac{\varepsilon}{c}
$$

which proves (5.9).

In view of $(1.5)$, any solution $u \in C\left(\mathbb{R} ; H^{1}(\mathbb{R})\right)$ of $(\mathrm{C}-\mathrm{H})$ satisfies $u_{t} \in C\left(\mathbb{R} ; L^{2}(\mathbb{R})\right)$ and thus belongs to $C^{1}\left(\mathbb{R} ; L^{2}(\mathbb{R})\right)$. This ensures that the mapping $t \mapsto x(t)=$ $\tilde{x}(u(t))$ is of class $C^{1}$ on $\mathbb{R}$. Setting $R(t, \cdot)=c \varphi(\cdot-x(t))$ and $w=u-R$ and differentiating (5.5) with respect to time we get

$$
\begin{aligned}
\int_{\mathbb{R}} w_{t}\left(\rho_{n_{0}} * \varphi\right)^{\prime}(\cdot-x(t)) & =\dot{x}(t) \int_{\mathbb{R}} w\left(\rho_{n_{0}} * \varphi\right)^{\prime \prime}(\cdot-x(t)) \\
& =-\dot{x}(t) \int_{\mathbb{R}} \partial_{x} w\left(\rho_{n_{0}} * \varphi\right)^{\prime}(\cdot-x(t)) \\
& =(\dot{x}(t)-c) O\left(\|w\|_{H^{1}}\right)+c O\left(\|w\|_{H^{1}}\right) .
\end{aligned}
$$

Substituting $u$ by $w+R$ in (1.5) and using that $R$ satisfies

$$
\partial_{t} R+(\dot{x}-c) \partial_{x} R+R \partial_{x} R+\left(1-\partial_{x}^{2}\right)^{-1} \partial_{x}\left(R^{2}+R_{x}^{2} / 2\right)=0,
$$

we infer that $w$ satisfies

$w_{t}-(\dot{x}-c) \partial_{x} R=-\frac{1}{2} \partial_{x}\left((w+R)^{2}-R^{2}\right)-\left(1-\partial_{x}^{2}\right)^{-1} \partial_{x}\left((w+R)^{2}-R^{2}+\frac{1}{2}\left(\left(w_{x}+R_{x}\right)^{2}-R_{x}^{2}\right)\right)$.

Taking the $L^{2}$-scalar product of this last equality with $\left(\rho_{n_{0}} * \varphi\right)^{\prime}(\cdot-x(t))$ and using (7.7) together with (5.9) we get

$$
\left|(\dot{x}-c)\left(\int_{\mathbb{R}} \partial_{x} R \partial_{x}\left(\rho_{n_{0}} * \varphi\right)(\cdot-x(t))+c O\left(\|w\|_{H^{1}}\right)\right)\right| \leq O\left(\|w\|_{H^{1}}\right) \lesssim K c \varepsilon_{0}
$$

and (7.4) leads, by taking $n_{0}$ large enough and possibly decreasing the value of $\varepsilon_{0}>0$ so that $K \varepsilon_{0} \ll 1$, to (5.7).

It remains to prove that (5.6) holds for $n_{0} \geq 0$ large enough. For this we notice that $\int_{\mathbb{R}} \varphi^{\prime} \varphi^{\prime}(\cdot-y)=(1-y) e^{-y}$ which ensures that for $n_{0} \geq 0$ large enough

$$
\frac{d}{d y} \int_{\mathbb{R}} \varphi\left(\rho_{n_{0}} * \varphi\right)^{\prime}(\cdot-y)=\int_{\mathbb{R}} \varphi^{\prime}\left(\rho_{n} * \varphi\right)^{\prime}(\cdot-y) \geq \frac{1}{4} e^{-\frac{1}{2}} \text { on }[-1 / 2,1 / 2] .
$$

Therefore $y \mapsto \int_{\mathbb{R}} \varphi\left(\rho_{n_{0}} * \varphi\right)^{\prime}(\cdot-y)$ is increasing on $[-1 / 2,1 / 2]$ and the proof is complete. 
7.3. Proof of Proposition 5.2. Let $u_{0} \in Y_{+}$satisfying (5.1) with $0<\varepsilon<\frac{c}{2^{8}}$. First we recall that, on account of (5.9) and Lemma 3.2, the solution $u$ to $(\mathrm{C}-\mathrm{H})$, emanating from $u_{0}$, satisfies (5.14)-(5.15) with $0 \leq \gamma \leq c$. Let $\left\{t_{n}\right\} \nearrow+\infty$. Since, by (5.7), $\left\{x\left(t_{n}+\cdot\right)-x\left(t_{n}\right)\right\}$ is uniformly equi-continuous, Arzela-Ascoli theorem ensures that there exists a subsequence $\left\{t_{n_{k}}\right\} \subset\left\{t_{n}\right\}$ and $\tilde{x} \in C(\mathbb{R})$ such that for all $T>0$,

$$
x\left(t_{n_{k}}+\cdot\right)-x\left(t_{n_{k}}\right) \underset{t \rightarrow+\infty}{\longrightarrow} \tilde{x} \text { in } C([-T, T]) .
$$

Now, since $u\left(t_{n}\right)$ is bounded in $Y_{+}$. There exists $\tilde{u}_{0} \in Y_{+}$and a subsequence of $\left\{t_{n_{k}}\right\}$ (that we still denote by $t_{n_{k}}$ to simplify the notation) such that

$$
\begin{aligned}
& u\left(t_{n_{k}}, \cdot+x\left(t_{n_{k}}\right)\right) \rightarrow \tilde{u}_{0} \text { in } H^{1}(\mathbb{R}) \\
& u\left(t_{n_{k}}, \cdot+x\left(t_{n_{k}}\right)\right) \rightarrow \tilde{u}_{0} \text { in } H_{l o c}^{1}(\mathbb{R}) \\
& y\left(t_{n_{k}}, \cdot+x\left(t_{n_{k}}\right)\right) \rightarrow * \quad \tilde{y}_{0}=\tilde{u}_{0}-\tilde{u}_{0, x x} \text { in } \mathcal{M}(\mathbb{R}) .
\end{aligned}
$$

Let $\tilde{u} \in C\left(\mathbb{R} ; Y_{+}\right)$be the solution to (1.5) emanating from $\tilde{u}_{0}$. On account of (7.8) and part 3. of Proposition 2.2 for any $t \in \mathbb{R}$,

$$
\begin{aligned}
& u\left(t_{n_{k}}+t, \cdot+x\left(t_{n_{k}}+t\right)\right) \rightarrow \tilde{u}(t, \cdot+\tilde{x}(t)) \text { in } H^{1}(\mathbb{R}), \\
& u\left(t_{n_{k}}+t, \cdot+x\left(t_{n_{k}}+t\right)\right) \rightarrow \tilde{u}(t, \cdot+\tilde{x}(t)) \text { in } H_{l o c}^{1}(\mathbb{R})
\end{aligned}
$$

Moreover, for any function $\phi \in C_{0}(\mathbb{R})$, it holds

$$
\left\langle y\left(t_{n_{k}}+t, \cdot+x\left(t_{n_{k}}+t\right)\right), \phi\right\rangle \rightarrow\langle\tilde{y}(t, \cdot+\tilde{x}(t)), \phi\rangle,
$$

where $\tilde{y}=\tilde{u}-\tilde{u}_{x x}$. Indeed, on one hand, it follows from part 3. of Proposition 2.2 that

$$
\left\langle y\left(t_{n_{k}}+t, \cdot+x\left(t_{n_{k}}\right)+\tilde{x}(t)\right), \phi\right\rangle \rightarrow\langle\tilde{y}(t, \cdot+\tilde{x}(t)), \phi\rangle
$$

and on the other hand, the uniform continuity of $\phi$ together with (7.8) ensure that

$$
\begin{aligned}
& \left\langle y\left(t_{n_{k}}+t, \cdot+x\left(t_{n_{k}}\right)+\tilde{x}(t)\right)-y\left(t_{n_{k}}+t, \cdot+x\left(t_{n_{k}}+t\right)\right), \phi\right\rangle \\
& =\left\langle y\left(t_{n_{k}}+t\right), \phi\left(\cdot-x\left(t_{n_{k}}\right)-\tilde{x}(t)\right)-\phi\left(\cdot-x\left(t_{n_{k}}+t\right)\right)\right\rangle \rightarrow 0
\end{aligned}
$$

In view of (7.10) we infer that $(\tilde{u}, \tilde{x}(\cdot))$ satisfies (5.5) and (5.9) with the same $\varepsilon$ than $(u, x(\cdot))$. Therefore, $(5.10)$ forces $(\tilde{u}, \tilde{x}(\cdot))$ to satisfy $(5.3)$ and the uniqueness result in Lemma 5.1 ensures that $\tilde{x}(\cdot)$ is a $C^{1}$-function and satisfies (5.7).

The proof of the $Y$-almost localization of the asymptotic object $\tilde{u}$ will now proceed by contradiction. Let us first explain it briefly. In the sequel, for $0<\gamma \leq \frac{2 c}{3}$ fixed, we call by $\mathrm{G}$ the conserved quantity

$$
G(u)=E(u)+\gamma\left\langle u-u_{x x}, 1\right\rangle .
$$

If $\tilde{u}$ is not $Y$-almost localized then $\tilde{u}$ loses a certain amount of $G$ close to $\tilde{x}(t)$ between 0 and some $T_{0}>0$. By the convergence results (7.11)-(7.12) we infer that for $n$ large enough, $u$ loses some fixed amount of $G$ close to $x(t)$ between $t_{n}$ and $t_{n}+T_{0}$. By the conservation of $G$ on the whole line and the almost monotonicity of $J_{\gamma, l}$ and $J_{\gamma, r}$ this ensures that for some $R>0$ and $\varepsilon_{0}>0$,

$$
J_{\gamma, l}^{R}\left(u\left(t_{n_{k}}+T_{0}, \cdot+x\left(t_{n_{k}}+T_{0}\right)\right)\right) \geq J_{\gamma, l}^{R}\left(u\left(t_{n_{k}}, \cdot+x\left(t_{n_{k}}\right)\right)\right)+\varepsilon_{0} .
$$


But by the almost monotonicity of $J_{\gamma, l}$, taking $\left\{t_{n_{k}}\right\} \subset\left\{t_{n}\right\}$ such that $n_{k} \geq n_{0}$ and $t_{n_{k+1}}-t_{n_{k}} \geq T_{0}$ we get

$$
J_{\gamma, l}^{R}\left(u\left(t_{n_{k}}, \cdot+x\left(t_{n_{k}}\right)\right)\right) \geq J_{\gamma, l}^{R}\left(u\left(t_{0}, \cdot+x\left(t_{0}\right)\right)\right)+k \varepsilon_{0} / 2
$$

which contradicts the conservation of $G$.

Let us now make this proof rigorously. For $v \in Y$ and $R>0$, we separate $G(v)$ into two parts :

$$
G_{o}^{R}(v)=\left\langle v^{2}+v_{x}^{2}+\gamma\left(v-v_{x x}\right), 1-\Psi(\cdot+R)+\Psi(\cdot-R)\right\rangle=J_{\gamma, r}^{R}(v)+J_{\gamma, l}^{R}(v),
$$

which almost "localizes" outside the ball of radius $R$ and

$$
G_{i}^{R}(v)=\left\langle v^{2}+v_{x}^{2}+\gamma\left(v-v_{x x}\right), \Psi(\cdot+R)-\Psi(\cdot-R)\right\rangle=G(v)-G_{o}^{R}(v),
$$

which almost "localizes" inside this ball. We first notice that it suffices to prove that for all $\varepsilon>0$, there exists $R_{\varepsilon}>0$ such that

$$
G_{o}^{R_{\varepsilon}}(\tilde{u}(t, \cdot+\tilde{x}(t)))<\varepsilon, \quad \forall t \in \mathbb{R} .
$$

Indeed if (7.13) is true for some $\left(\varepsilon, R_{\varepsilon}\right)$ then $(\tilde{u}, \tilde{x})$ satisfies $(1.7)$ with $\left(\varepsilon / 2,2 R_{\varepsilon}\right)$. As indicated above, we prove (7.13) by contradiction. Assuming that (7.13) is not true, there exists $\varepsilon_{0}>0$ such that for any $R>0$ there exists $t_{R} \in \mathbb{R}$ satisfying

$$
G_{o}^{R}\left(\tilde{u}\left(t_{R}, \cdot+\tilde{x}\left(t_{R}\right)\right)\right) \geq \varepsilon_{0}
$$

Let $R_{0}>0$ such that

$$
G_{o}^{R_{0}}(\tilde{u}(0)) \leq \frac{\varepsilon_{0}}{10}
$$

and $K_{0} e^{-R_{0} / 6}<\frac{\varepsilon_{0}}{10}$. The conservation of $G$ then forces

$$
G_{i}^{R_{0}}\left(\tilde{u}\left(t_{R_{0}} \cdot+\tilde{x}\left(t_{R_{0}}\right)\right) \leq G_{i}^{R_{0}}(\tilde{u}(0))-\frac{9}{10} \varepsilon_{0} .\right.
$$

Noticing that $\Psi(\cdot+R)-\Psi(\cdot-R) \in C_{0}(\mathbb{R})$, the convergence results (7.11)-(7.12) ensure that for $k \geq k_{0}$ with $k_{0}$ large enough,

$$
G_{i}^{R_{0}}\left(u\left(t_{n_{k}}+t_{R_{0}} \cdot \cdot+x\left(t_{n_{k}}+t_{R_{0}}\right)\right)\right) \leq G_{i}^{R_{0}}\left(u\left(t_{n_{k}}, \cdot+x\left(t_{n_{k}}\right)\right)\right)-\frac{4}{5} \varepsilon_{0} .
$$

We first assume that $t_{R_{0}}>0$. By (5.14)-(5.15) and the conservation of $G$ this ensures that

$$
J_{\gamma, l}^{R_{0}}\left(u\left(t_{n_{k}}+t_{R_{0}} \cdot \cdot+x\left(t_{n_{k}}+t_{R_{0}}\right)\right)\right) \geq J_{\gamma, l}^{R_{0}}\left(u\left(t_{n_{k}}, \cdot+x\left(t_{n_{k}}\right)\right)\right)+\frac{7}{10} \varepsilon_{0} .
$$

Now we take a subsequence $\left\{t_{n_{k}^{\prime}}\right\}$ of $\left\{t_{n_{k}}\right\}$ such that $t_{n_{k+1}^{\prime}}-t_{n_{k}^{\prime}} \geq t_{R_{0}}$ and $n_{k}^{\prime} \geq n_{k_{0}}$. From (7.16) and again (5.15), we get that for any $k \geq 0$,

$$
J_{\gamma, l}^{R_{0}}\left(u\left(t_{n_{k}^{\prime}}, \cdot+x\left(t_{n_{k}^{\prime}}\right)\right)\right) \geq J_{\gamma, l}^{R_{0}}\left(u\left(t_{n_{0}^{\prime}}, \cdot+x\left(t_{n_{0}^{\prime}}\right)\right)\right)+\frac{3}{5} k \varepsilon_{0} \underset{k \rightarrow+\infty}{\longrightarrow}+\infty
$$

that contradicts the conservation of $G$ and thus proves the $Y$-almost localization of $\tilde{u}$. Finally, if $t_{R_{0}}<0$, then for $k \geq k_{0}$ such that $t_{n_{k}}>\left|t_{R_{0}}\right|$ we get in the same way

$$
J_{\gamma, r}^{R_{0}}\left(u\left(t_{n_{k}}, \cdot+x\left(t_{n_{k}}\right)\right)\right) \leq J_{\gamma, r}^{R_{0}}\left(u\left(t_{n_{k}}-\left|t_{R_{0}}\right|, \cdot+x\left(t_{n_{k}}-\left|t_{R_{0}}\right|\right)\right)\right)-\frac{7}{10} \varepsilon_{0} .
$$


As above, this implies the existence of a subsequence $\left\{t_{n_{k}^{\prime}}\right\}$ of $\left\{t_{n_{k}}\right\}$ such that

$$
J_{\gamma, r}^{R_{0}}\left(u\left(t_{n_{k}^{\prime}} \cdot+x\left(t_{n_{k}^{\prime}}\right)\right)\right) \leq J_{\gamma, r}^{R_{0}}\left(u\left(t_{n_{0}^{\prime}} \cdot+x\left(t_{n_{0}^{\prime}}\right)\right)\right)-\frac{3}{5} k \varepsilon_{0} \underset{k \rightarrow+\infty}{\longrightarrow}-\infty .
$$

which also leads to a contradiction.

Acknowledgements : The author is very grateful to Professor Yvan Martel for his encouragements, his careful reading of a first version of the manuscript and having suggested the proof of Lemma 6.3. He also thank Professor Masaya Maeda for pointing out a flaw in the version of Lemma 5.1 stated in [21] as well as the anonymous Referees for valuable remarks. Finally, the author gratefully acknowledges the hospitality and support of IHES, where part of this work was done during the program on nonlinear waves in spring 2016.

Conflict of Interest : The author declares that he has no conflict of interest.

\section{REFERENCES}

[1] B. Alvarez-Samaniego and D. Lannes, Large time existence for 3D water-waves and asymptotics, Invent. Math. 171 (2009), 165-186.

[2] R. Beals, D.H. Sattinger and J. Szmigielski, Multi-peakons and the classical moment problem, Adv. Math. 154 (2000), no. 2, 229-25\%.

[3] T. B. Benjamin, The stability of solitary waves. Proc. Roy. Soc. London Ser. A 328 (1972), $153-183$.

[4] N. Bourbaki, Eléments de Mathématique, Intégration, Chapitre 9, Herman Paris 1969.

[5] A. Bressan, G. Chen, And Q. Zhang, Uniqueness of conservative solutions to the CamassaHolm equation via characteristics, Discr. Cont. Dyn. Syst. 35 (2015), 25?42.

[6] A. Bressan and A. Constantin, Global conservative solutions of the Camassa-Holm equation, Arch. Rational Mech. Anal. 187 (2007), 215-239.

[7] A. Bressan And A. Constantin, Global dissipative solutions of the Camassa-Holm equation, Analysis and Applications 5 (2007), 1-27.

[8] H. Cai, G. Chen, R.M. Chen And Y. Shen, Lipschitz metric for the Novikov equation, arXiv:1611.08277.

[9] R. Camassa And D. Holm, An integrable shallow water equation with peaked solitons, Phys. rev. Lett. 71 (1993), 1661-1664.

[10] R. Camassa, D. Holm and J. Hyman, An new integrable shallow water equation, Adv. Appl. Mech. 31 (1994).

[11] A. Constantin , Existence of permanent and breaking waves for a shallow water equations: a geometric approach, Ann. Inst. Fourier 50 (2000), 321-362.

[12] A. Constantin, On the scattering problem for the Camassa-Holm equation, Proc. Roy. Soc. London Ser. A . 457 (2001), 953-970.

[13] A. Constantin And J. Escher, Global existence and blow-up for a shallow water equation, Annali Sc. Norm. Sup. Pisa 26 (1998), 303-328.

[14] A. Constantin, V. Gerdjikov and R. Ivanov, Inverse scattering transform for the CamassaHolm equation, Inverse problems 22 (2006), 2197-220\%.

[15] A. Constantin and D. Lannes, The hydrodynamical relevance of the Camassa-Holm and Degasperis-Procesi Equations Arch. Rat. Mech. Anal., 192 (2009), 165-186.

[16] A. Constantin And B. Kolev, Geodesic flow on the diffeomorphism group of the circle, Comment. Math. Helv. 78 (2003), 787-804.

[17] A. Constantin And L. Molinet, Global weak solutions for a shallow water equation, Comm. Math. Phys. 211 (2000), 45-61.

[18] A. Constantin And W. Strauss, Stability of peakons, Commun. Pure Appl. Math. 53 (2000), 603-610.

[19] J. EckHARDt, AND G.Teschl On the isospectral problem of the dispersionless Camassa-Holm equation, Adv. Math. 235 (2013), 469-495.

[20] K. El Dika and Y. Martel, Stability of $N$ solitary waves for the generalized BBM equations, Dyn. Partial Differ. Equ. 1 (2004), 401-437. 
[21] K. El Dika and L. Molinet, Stability of multipeakons, Ann. Inst. H. Poincaré Anal. Non Linéaire 26 (2009), no. 4, 1517-1532.

[22] K. El Dika And L. Molinet, Stability of train of anti-peakons -peakons, Discrete Contin. Dyn. Syst. Ser. B 12 (2009), no. 3, 561-577.

[23] M. Grillakis, J. Shatah and W. Strauss, Stability theory of solitary waves in the presence of symmetry, J. Funct. Anal. 74 (1987), 160-197.

[24] D. Iftimie, Large time behavior in perfect incompressible flows, Partial differential equations and applications,119-179, Sémin. Congr., 15, Soc. Math. France, Paris, $200 \%$.

[25] R.S. Johnson, Camassa-Holm, Korteweg-de Vries and related models for water waves, J. Fluid Mech. 455 (2002), 63-82.

[26] B. Kolev, Lie groups and mechanics: an introduction, J. Nonlinear Math. Phys. 11 (2004), 480-498.

[27] B. Kolev, Poisson brackets in hydrodynamics, Discrete Contin. Dyn. Syst. 19 (2007), 555574.

[28] Y. Martel, F. Merle and T-P. Tsai Stability and asymptotic stability in the energy space of the sum of $N$ solitons for subcritical gKdV equations. Comm. Math. Phys. 231 (2002), $347-373$.

[29] Y. Martel and F. Merle Asymptotic stability of solitons for subcritical generalized KdV equations. Arch. Ration. Mech. Anal. 157 (2001), no. 3, 219-254.

[30] Y. Martel and F. Merle Asymptotic stability of solitons of the gKdV equations with general nonlinearity. Math. Ann. 341 (2008), no. 2, 391-42\%.

[31] L. Molinet On well-posedness results for Camassa-Holm equation on the line: a survey. $J$. Nonlinear Math. Phys. 11 (2004), 521-533.

Luc Molinet, Institut Denis Poisson, Université de Tours, Université d'Orléans, CNRS, Parc Grandmont, 37200 Tours, France.

E-mail address: Luc.Molinet@univ-tours.fr 